\title{
LATTICES ON PARABOLIC TREES
}

\author{
Lisa Carbone and Dennis Clark
}

\begin{abstract}
Let $X$ be a locally finite tree, and let $G=\operatorname{Aut}(X)$. Then $G$ is a locally compact group. A non-uniform $X$-lattice is a discrete subgroup $\Gamma \leq G$ such that the quotient graph of groups $\Gamma \backslash \backslash X$ is infinite but has finite covolume, and a non-uniform G-lattice is a discrete subgroup $\Lambda$ such that $\Lambda \backslash G$ is not compact yet has a finite $G$-invariant measure. We show that if $X$ has a unique end and if $G$ contains a non-uniform $X$-lattice, then $G$ contains a non-uniform $G$-lattice if and only if any path directed towards the end of the edge-indexed quotient of $X$ has unbounded index.
\end{abstract}

\section{Notation and main results}

Let $X$ be a locally finite tree and $G=\operatorname{Aut}(X)$. Then $G$ is naturally a locally compact group with compact open vertex stabilizers $G_{x}, x \in V X([\mathrm{BL}],(3.1))$. A subgroup $\Gamma \leq G$ is discrete if and only if $\Gamma_{x}$ is a finite group for some (hence for every) $x \in V X$.

Let $\mu$ be a (left) Haar measure on $G$. By a G-lattice $\Gamma$ we mean a discrete subgroup $\Gamma \leq G=\operatorname{Aut}(X)$ such that $\Gamma \backslash G$ has a finite measure $\mu(\Gamma \backslash G)$. We call $\Gamma$ a uniform $G$ lattice if $\Gamma \backslash G$ is compact, and a non-uniform $G$-lattice if $\Gamma \backslash G$ is not compact yet $\mu(\Gamma \backslash G)$ is finite.

A discrete subgroup $\Gamma \leq G$ is called an $X$-lattice if

$$
\operatorname{Vol}(\Gamma \backslash \backslash X):=\sum_{x \in V(\Gamma \backslash X)} \frac{1}{\left|\Gamma_{x}\right|}
$$

is finite, a uniform $X$-lattice if $\Gamma \backslash X$ is a finite graph, and a non-uniform lattice if $\Gamma \backslash X$ is infinite but $\operatorname{Vol}(\Gamma \backslash \backslash X)$ is finite.

When $G$ is unimodular, $\mu\left(G_{x}\right)$ is constant on $G$-orbits, so we can define ([BL], (1.5)):

$$
\mu(G \backslash \backslash X) \quad:=\sum_{x \in V(G \backslash X)} \frac{1}{\mu\left(G_{x}\right)} .
$$

The first author was supported in part by NSF grant \#DMS-9800604. The second author was supported by a VIGRE summer research grant for undergraduates at Harvard University.

2000 Mathematics subject classification. Primary 20F32; secondary 22F50.

Typeset by $\mathcal{A}_{\mathcal{M}} \mathcal{S}-\mathrm{T}_{\mathrm{E}} \mathrm{X}$ 
(0.1) Theorem ([BL], (1.6)). For a discrete subgroup $\Gamma \leq G=A$ ut $(X)$, the following conditions are equivalent:

(a) $\Gamma$ is an $X$-lattice, that is, $\operatorname{Vol}(\Gamma \backslash \backslash X)<\infty$.

(b) $\Gamma$ is a $G$-lattice (hence $G$ is unimodular), and $\mu(G \backslash \backslash X)<\infty$.

In this case:

$$
\operatorname{Vol}(\Gamma \backslash \backslash X)=\mu(\Gamma \backslash G) \cdot \mu(G \backslash \backslash X) .
$$

In $[\mathrm{BCR}]$ we prove the 'Lattice Existence Theorem', namely that $G$ contains an $X$ lattice if and only if $G$ is unimodular and $\mu(G \backslash \backslash X)<\infty$. In particular, it is shown in [BCR] that if $G$ is unimodular, $\mu(G \backslash \backslash X)<\infty$, and $G \backslash X$ is infinite, then $G$ contains a (necessarily non-uniform) $X$-lattice $\Gamma$, which is a uniform $G$-lattice. In [CR1], we show that if $X$ has more than one end, and if $G$ contains a non-uniform $X$-lattice, then $G$ contains a non-uniform $G$-lattice. Here our main result is the following:

(0.2) Theorem. Let $X$ be a locally finite tree and let $G=\operatorname{Aut}(X)$. If $X$ has a unique end and if $G$ contains a non-uniform $X$-lattice, then $G$ contains a non-uniform $G$-lattice if and only if any path directed towards the end of the edge-indexed quotient graph has unbounded index.

Let $\Gamma$ be a non-uniform $X$-lattice. Then the diagram of natural projections

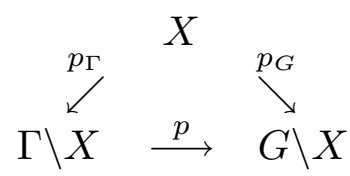

commutes. By Theorem $(0.1), \Gamma$ is a $G$-lattice. To determine if $\Gamma$ is uniform or nonuniform in $G$, we use the following:

(0.3) Lemma ([BL], (1.5) (8)). Let $x \in V X$. The following conditions are equivalent:

(a) $\Gamma$ is a uniform G-lattice.

(b) Some fiber $p^{-1}\left(p_{G}(x)\right) \cong \Gamma \backslash G / G_{x}$ is finite.

(c) Every fiber of $p$ is finite.

It follows that if $G \backslash X$ is finite, then $\Gamma$ is a uniform (respectively non-uniform) $X$ lattice if and only if $\Gamma$ is a uniform (respectively non-uniform) $G$-lattice. Conversely, the assumption that $X$ has a unique end implies that $G \backslash X$ is infinite. To construct a non-uniform $G$-lattice, our task is to construct a discrete group $\Gamma$ with $\Gamma \backslash X$ infinite, $\operatorname{Vol}(\Gamma \backslash \backslash X)<\infty$, and some (hence every) fiber of the projection $p$ infinite.

Locally finite trees with a unique end are called parabolic ([BL], Ch 9$)$. Let $(A, i)$ be an edge-indexed graph in the sense of ([BL], Ch 1$)$. We say that $(A, i)$ is parabolic if $X=\widehat{(A, i)}$ is a parabolic tree.

Theorem (0.2) will be deduced from the following result about edge-indexed graphs. Here we follow the notations and terminology of Section 1. 
(0.4) Theorem. Let $(A, i)$ be a parabolic tree with finite volume. Then there is a covering $p:(B, j) \longrightarrow(A, i)$ of edge-indexed graphs with infinite fibers such that $(B, j)$ has finite volume if and only if $(A, i)$ contains a ray with unbounded index.

As a corollary of Theorem (0.4) we have the following:

(0.5) Theorem. Let $(A, i)$ be an infinite parabolic tree with finite volume. Then there exists a (necessarily non-uniform) $X$-lattice $\Gamma \leq G_{(A, i)}$ which is a non-uniform $G_{(A, i)^{-}}$ lattice if and only if $(A, i)$ contains a ray with unbounded index.

(0.6) Corollary. Let $X$ be a locally finite parabolic tree, $G=\operatorname{Aut}(X), \mu$ a (left) Haar measure on $G$, and $H \leq G$ a unimodular closed subgroup acting without inversions with projection $p_{H}: X \longrightarrow A=H \backslash X$, and edge-indexed quotient $(A, i)=I(H \backslash \backslash X)$. Assume that $H=G_{(A, i)}$ and that $\mu(H \backslash \backslash X)<\infty$. If $X$ has a unique end, and $H \backslash X$ is infinite, then there exists a (necessarily non-uniform) $X$-lattice $\Gamma \leq H$ which is a non-uniform $H$-lattice if and only if any path directed towards the end of the edge-indexed quotient graph of $X$ has unbounded index.

We call a lattice on a parabolic tree $X$ a parabolic $X$-lattice. By ([BL], Ch 9), for $x_{0} \in V X$, a parabolic lattice $\Gamma$ is the infinite ascending union of the vertex stabilizers $\Gamma_{x}$ as $x$ approaches the end $\epsilon$ of $X$ along the unique path from $x_{0}$.

The following gives an infinite tower of coverings with infinite fibers and finite volume over an edge-indexed graph that admits a lattice:

(0.7) Theorem. Let $(A, i)$ be a parabolic tree with finite volume. If $(A, i)$ has a covering $p:(B, j) \longrightarrow(A, i)$ with infinite fibers and finite volume, then $(A, i)$ has an infinite sequence of coverings:

$$
\left(B_{0}, j_{0}\right) \stackrel{p_{0}}{\longrightarrow}\left(B_{1}, j_{1}\right) \stackrel{p_{1}}{\longrightarrow}\left(B_{2}, j_{2}\right) \stackrel{p_{2}}{\longrightarrow} \ldots \quad \longrightarrow \quad(A, i)
$$

with infinite fibers, and there exists $a_{0} \in V A, b_{l} \in V B_{l}$ with $p_{0}\left(b_{l}\right)=a_{0}$ for $l=1,2, \ldots$ such that

$$
\operatorname{Vol}_{b_{l}}\left(B_{l}, j_{l}\right) \longrightarrow \operatorname{Vol}_{a_{0}}(A, i)<\infty, \text { as } l \longrightarrow \infty .
$$

Hence we obtain an infinite ascending chain of closed subgroups of Aut $(X)$ :

$$
G_{\left(B_{0}, j_{0}\right)} \leq G_{\left(B_{1}, j_{1}\right)} \leq G_{\left(B_{2}, j_{2}\right)} \leq \ldots \leq G_{(A, i)},
$$

(with notation as in (1.5)), and non-uniform $G_{(A, i)}$-lattices $\Gamma_{l}$ with $\Gamma_{l} \leq G_{\left(B_{l}, j_{l}\right)}$, $l=0,1,2 \ldots$

In Section 1, we outline the basics of parabolic edge-indexed graphs and a method for constructing (parabolic) $X$-lattices. In Section 2, we prove Theorem (0.4) in the case that $(A, i)$ is a parabolic ray. In Section 3, we prove Theorem (0.4). In Section 4, we prove Theorem (0.7). 


\section{Constructing parabolic $X$-lattices}

Let $(A, i)$ be an edge-indexed graph in the sense of ([BL], Ch 2$)$. We say that $(A, i)$ is parabolic if $X=\widetilde{(A, i)}$ is a parabolic tree (as in ([BL], Section 9$)$ ), that is $X$ has a unique end denoted $\epsilon$. It follows that $(A, i)$ is an infinite tree with a unique end denoted $\epsilon_{A}$. Moreover, in $(A, i)$ we have

$$
i(e)=1 \text { for every } e \in E A \text { directed towards } \epsilon_{A} \text {. }
$$

We choose $e \in E A$ to be positively oriented if $e$ is directed towards the unique end $\epsilon_{A}$ of $(A, i)$.

A parabolic edge-indexed tree $(A, i)$ is automatically unimodular in the sense of ([BL], (2.6)), and has bounded denominators in the sense of $([\mathrm{BL}],(2.6))$. If follows $([\mathrm{BK}],(2.5))$ that a parabolic edge-indexed tree $(A, i)$ automatically admits a finite (faithful) grouping; that is, there is a graph of finite groups $\mathbb{A}=(A, \mathcal{A})$ such that $i(e)=\left[\mathcal{A}_{\partial_{0} e}: \alpha_{e} \mathcal{A}_{e}\right]$ for every $e \in E A$, where $\alpha_{e}: \mathcal{A}_{e} \hookrightarrow \mathcal{A}_{\partial_{0} e}$.

Let $(A, i)$ be a parabolic edge-indexed tree. For $e \in E A$, we put

$$
\Delta(e)=\frac{i(\bar{e})}{i(e)}
$$

For an edge path $\gamma=\left(e_{1}, \ldots, e_{n}\right)$ in $A$, we put $\Delta(\gamma)=\Delta\left(e_{1}\right) \ldots \Delta\left(e_{n}\right)$. Fix $a_{0} \in V A$,

and let $\gamma$ be the unique path from $a_{0}$ to $a \in V A$. We denote $\Delta(\gamma)$ by $\frac{\Delta a}{\Delta a_{0}}$. Following ([BL], (2.6)), we define the volume of $(A, i)$ at $a_{0} \in V A$ :

$$
\operatorname{Vol}_{a_{0}}(A, i)=\sum_{a \in V A} \frac{1}{\left(\frac{\Delta a}{\Delta a_{0}}\right)} .
$$

For $a_{1} \in V A$, we have $([\mathrm{BL}],(2.6))$ :

$$
V o l_{a_{1}}(A, i)=\frac{\Delta a_{1}}{\Delta a_{0}} \operatorname{Vol}_{a_{0}}(A, i)
$$

so the condition

$$
\operatorname{Vol}(A, i)<\infty
$$

defined by $\operatorname{Vol}_{a_{0}}(A, i)<\infty$, is independent of the choice of $a_{0}$.

It follows that if $(A, i)$ is a parabolic edge-indexed tree of finite volume, then $(A, i)$ admits a finite (faithful) grouping $\mathbb{A}$ of finite volume, where

$$
\operatorname{Vol}(\mathbb{A})=\frac{1}{\left|\mathcal{A}_{a_{0}}\right|} \operatorname{Vol}_{a_{0}}(A, i)
$$


Hence $(A, i)$ admits a non-uniform $X$-lattice $\Gamma=\pi_{1}\left(\mathbb{A}, a_{0}\right)$ for $a_{0} \in V A$. We call a parabolic edge-indexed tree of finite volume a parabolic lattice tree.

A covering of edge-indexed graphs $([\mathrm{BL}],(2.5))$

$$
p:(B, j) \longrightarrow(A, i)
$$

is a graph morphism $p: B \longrightarrow A$ such that for all $e \in E A$ with $\partial_{0}(e)=a$ and $b \in p^{-1}(a)$, we have

$$
i(e)=\sum_{f \in p_{(b)}^{-1}(e)} j(f)
$$

where $p_{(b)}: E_{0}^{B}(b) \longrightarrow E_{0}^{A}(a)$ is the local map on stars $E_{0}^{B}(b)$ and $E_{0}^{A}(a)$ of vertices $b \in V B$ and $a \in V A$ (cf. [BL], (2.5)). If $b \in V B, p(b)=a \in V A$, then we can identify

$$
(\widetilde{A, i, a})=X=(\widetilde{B, j, b})
$$

so that the diagram of natural projections

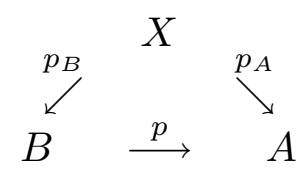

commutes.

Given $(A, i)$ let $X=\widetilde{(A, i)}$ with projection $p_{(A, i)}: X \longrightarrow A$. Let $G=\operatorname{Aut}(X)$ and let

$$
G_{(A, i)}:=\quad\left\{g \in G \mid p_{(A, i)} \circ g=p_{(A, i)}\right\} .
$$

Then $G_{(A, i)}$ is a closed subgroup of $G=\operatorname{Aut}(X)$ ([BL], (3.3)).

If $(A, i)$ is a parabolic lattice tree and $\mathbb{A}$ is a finite faithful grouping of $(A, i)$ of finite volume, then ([BL], (3.3)) for $a_{0} \in V A$ we have:

$$
\Gamma=\pi_{1}\left(\mathbb{A}, a_{0}\right) \leq G_{(A, i)} .
$$

Moreover, if $p:(B, j) \longrightarrow(A, i)$ is a covering, then:

(a) $G_{(B, j)} \leq G_{(A, i)}$.

(b) For $a_{0} \in V A, b_{0} \in V B$ with $p\left(b_{0}\right)=a_{0}$, we have the Bass-Rosenberg volume formula $([\mathrm{R}])$ :

$$
\operatorname{Vol}_{b_{0}}(B, j)=\operatorname{Vol}_{a_{0}}(A, i) \times \operatorname{Vol}_{(B, j)}\left(p^{-1}\left(a_{0}\right)\right),
$$

where

$$
\operatorname{Vol}_{(B, j)}\left(p^{-1}\left(a_{0}\right)\right)=\sum_{b \in p^{-1}\left(a_{0}\right)} \frac{1}{\left(\frac{\Delta b}{\Delta b_{0}}\right)} .
$$




\section{Parabolic lattice rays}

In this section, we prove Theorem $(0.4)$ in the case that $(A, i)$ is a parabolic lattice ray.

(2.1) We recall that if $(A, i)$ is a parabolic edge-indexed tree, then $i(e)=1$ for every edge $e$ directed towards the end $\epsilon_{A}$ of $(A, i)$.

(2.2) Definition. A parabolic lattice ray $(A, i)$ is an edge-indexed graph of the form:

$(A, i)=$

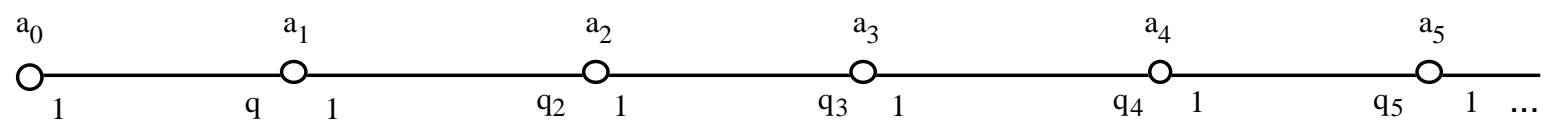

with almost all $q_{k} \geq 2$.

We shall refer to the terminal vertex $a_{0}$ as the initial vertex of $(A, i)$.

Let $(A, i)$ be a parabolic lattice ray, let $X=\widetilde{(A, i)}$, and let $G=A u t(X)$. Then $(A, i)$ has a finite grouping of finite volume and hence gives rise to a non-uniform $X$-lattice. We seek a covering $p:(B, j) \rightarrow(A, i)$ such that $(B, j)$ has infinite fibers and finite volume. This will give rise to a non-uniform $G$-lattice.

(2.3) Lemma. Let $(A, i)$ be a parabolic lattice ray. Then any covering $p:(B, j) \longrightarrow$ $(A, i)$ of edge-indexed graphs is an edge-indexed parabolic tree.

Proof. Let $p:(B, j) \longrightarrow(A, i)$ be a covering. It is clear that $(B, j)$ is a tree. Moreover, for $a_{k} \in V A$, the local fiber above

$$
\left(E_{0}^{A}\left(a_{k}\right), i\right)=
$$

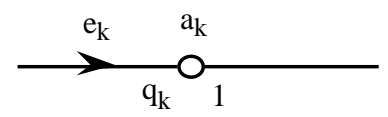

looks like:

$$
\left(E_{0}^{B}\left(b_{k}\right), j\right)=
$$

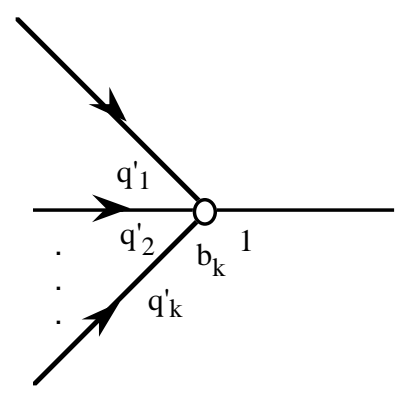


where $p\left(b_{k}\right)=a_{k}$ and since $p:(B, j) \longrightarrow(A, i)$ is a covering, we have

$$
q_{1}^{\prime}+q_{2}^{\prime}+\cdots+q_{n}^{\prime}=q_{k}
$$

Furthermore, since $p$ is a covering, for every $f \in p^{-1}\left(e_{k}\right)$ we must have $j(f)=1$ for every $k=1,2, \ldots$ Any vertex $b_{0}$ in the fiber $p^{-1}\left(a_{0}\right)$ above $a_{0}$ must be a terminal vertex. It follows that any infinite reduced path from $b_{0}$ is a sequence $\left(f_{1}, f_{2}, f_{3}, \ldots\right)$ of edges with $j\left(f_{k}\right)=1$ for each $k$. Hence $(B, j)$ is parabolic.

\section{(2.4) Coverings of parabolic lattice rays.}

Let $(A, i)$ be a parabolic lattice ray. Let $p:(B, j) \longrightarrow(A, i)$ be a covering of edgeindexed graphs. It follows from Lemma $(2.3)$ that $(B, j)$ is of the form:

$(B, j)=$

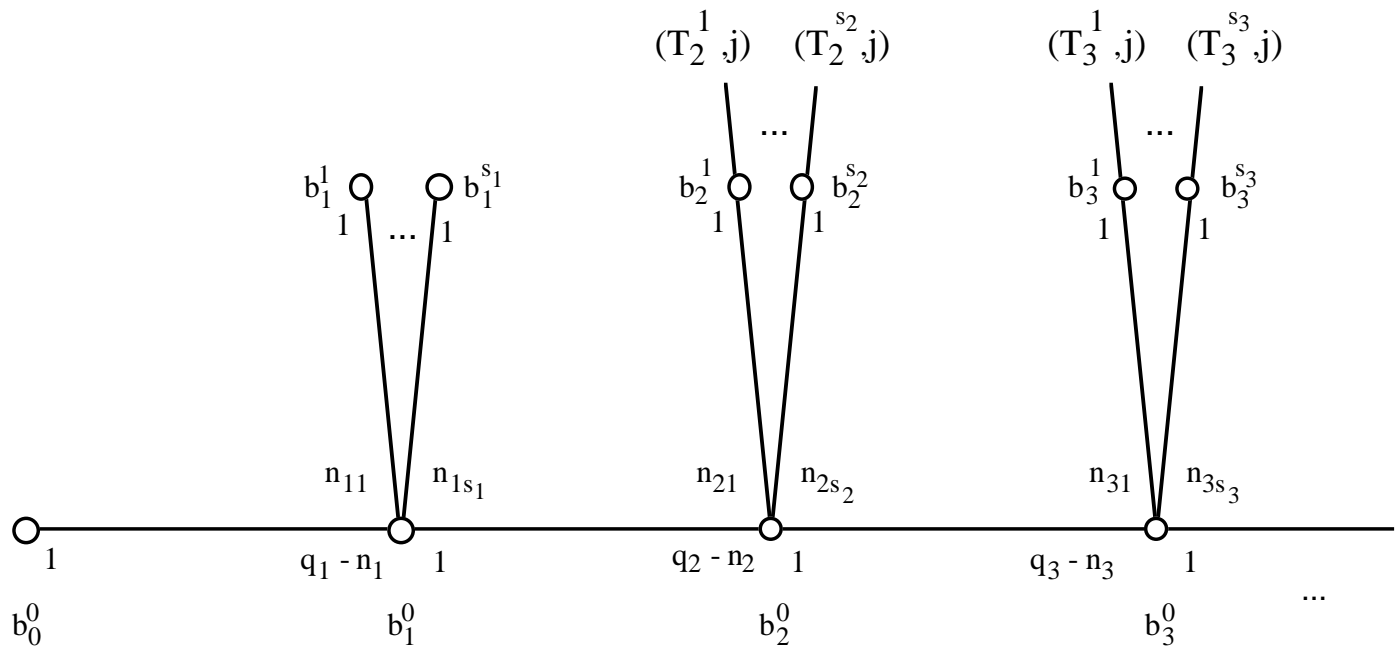

where

$$
\begin{gathered}
n_{k} \geq 0 \text { for each } k \geq 1, \\
n_{k 1}+\cdots+n_{k s_{k}}=n_{k} \text { for each } k \geq 1,
\end{gathered}
$$

and we have chosen a 'base-ray' in $(B, j)$, say with vertex sequence $b_{0}^{0}, b_{1}^{0}, \ldots$ For each $k \geq 1$, let $b_{k}^{1}, \ldots, b_{k}^{s_{k}}$ be the vertices at distance 1 from $b_{k}^{0}$ other than $b_{k-1}^{0}$ and $b_{k+1}^{0}$. For $k \geq 2$, to each vertex $b_{k}^{l_{k}}, l_{k}=1, \ldots, s_{k}$ is attached a finite (possibly empty) 'dominantrooted edge-indexed tree', denoted $\left(T_{k}^{l_{k}}, j\right)$. (For $l_{1}=1, \ldots, s_{1},\left(T_{1}^{l_{1}}, j\right)$ is necessarily empty since $b_{1}^{l_{1}}$ is necessarily a terminal vertex), where 'dominant-rooted' is defined as follows: Let $(T, j)$ be a finite edge-indexed tree, and suppose that $(T, j)$ is attached to an edge-indexed graph $(A, i)$ at a vertex $v \in V T \cap V A$. We will refer to the vertex $v$ as the root of $(T, j)$. We call $(T, j)$ dominant-rooted if all edges pointing towards the root have index 1. 
Let $v \in V T$. The height $h t(v)$ of $v$ in $T$ is defined to be the length of the (unique) redeuced path in $T$ from the root $v_{0}$ to $v$. Let

$$
k=\max _{v \in V T} h t(v) .
$$

For each vertex $x$ at height $1 \leq s \leq k$, let $\Sigma_{s}^{x}$ be the sum of the indices of edges emanating from $x$.

Let $c_{1}, \ldots, c_{k} \in \mathbb{Z}_{>0}$. We say that a finite edge-indexed tree $(T, j)$ is $\left(c_{1}, \ldots, c_{k}\right)$ regular if the following conditions hold:

(a) $(T, j)$ is dominant rooted.

(b) All terminal vertices in $V T-\left\{v_{0}\right\}$ have height $k$.

(c) For all vertices $x$ at height $s, 1 \leq s \leq k$, we have:

$$
\Sigma_{s}^{x}=c_{s} .
$$

(2.5) Lemma. Let $c_{1}, \ldots, c_{k} \in \mathbb{Z}_{>0}$ and let $(T, j)$ be a $\left(c_{1}, \ldots, c_{k}\right)$-regular tree with root $v_{0}$. Then:

$$
\operatorname{Vol}_{v_{0}}(T, j)=1+\sum_{s=1}^{k-1} \prod_{l=0}^{s-1} c_{k-l-1}
$$

for each $k \geq 2$.

Proof. We use induction on $k \geq 2$. For $k=2$, we have: $\left(T_{2}, j\right)=$

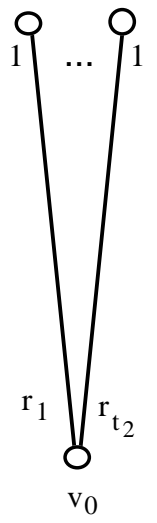

for some $r_{1}, \ldots, r_{t_{2}} \quad$ with

$$
r_{1}+\cdots+r_{t_{2}}=c_{1} .
$$


Then

$$
\begin{aligned}
\operatorname{Vol}_{v_{0}}\left(T_{2}, j\right) & =1+r_{1}+\cdots+r_{t_{2}} \\
& =1+c_{1} \\
& =1+\sum_{s=1}^{1} \prod_{l=0}^{s-1} c_{2-l-1} .
\end{aligned}
$$

Assume that for some $k>3$ and for each $\left(c_{1}, \ldots, c_{k-1}\right)$-regular tree $\left(T_{k-1}, j\right)$ we have

$$
V_{k-1}:=\operatorname{Vol}_{v_{0}}\left(T_{k-1}, j\right)=1+\sum_{s=1}^{k-2} \prod_{l=0}^{s-1} c_{(k-1)-l-1}
$$

Then

$\left(T_{k}, j\right)=$

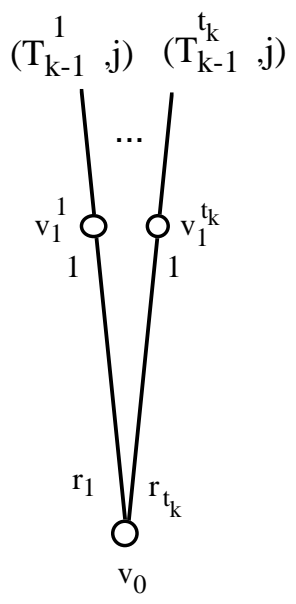

where $\left(T_{k-1}^{1}, j\right), \ldots,\left(T_{k-1}^{t_{k}}, j\right)$ are $\left(c_{1}, \ldots, c_{k-1}\right)$-regular trees and therefore (by induction) have volume $V_{k-1}$.

Then

$$
r_{1}+\cdots+r_{t_{k}}=c_{k-1}
$$

thus

$$
\begin{aligned}
V_{k}:=\operatorname{Vol}_{v_{0}}\left(T_{k}, j\right) & \stackrel{(1)}{=} 1+r_{1} V_{k-1}+\cdots+r_{t_{k}} V_{k-1} \\
& =1+V_{k-1}\left(r_{1}+\cdots+r_{t_{k}}\right) \\
& =1+V_{k-1} c_{k-1} \\
& \stackrel{(1)}{=} 1+c_{k-1}\left[1+\sum_{s=1}^{k-2} \prod_{l=0}^{s-1} c_{(k-1)-l-1}\right] \\
& =1+\sum_{s=1}^{k-1} \prod_{l=0}^{s-1} c_{k-l-1} . \square
\end{aligned}
$$


As a corollary, we observe that the trees $\left(T_{n}^{m}, j\right)$ of $(B, j)$ as in $(2.4)$ satisfy the hypothesis of Lemma $(2.5)$ for $\left(c_{1}, \ldots, c_{n}\right)=\left(q_{1}, \ldots, q_{n}\right)$, and therefore have volume

$$
1+\sum_{s=1}^{k-1} \prod_{l=0}^{s-1} q_{k-l-1}
$$

(2.6) Corollary. Let $(A, i)$ be a parabolic lattice ray, let $p:(B, j) \longrightarrow(A, i)$ be the covering as in (2.4) 'Coverings of parabolic lattice rays', and let $V_{k}$ be as in Lemma (2.5). Then

$$
\begin{aligned}
\operatorname{Vol}_{b_{0}^{0}}(B, j) & =1+\sum_{k=1}^{\infty} \frac{\left[1+V_{k}\left(n_{k 1}+\cdots+n_{k s_{k}}\right)\right]}{\left(q_{1}-n_{1}\right)\left(q_{2}-n_{2}\right) \ldots\left(q_{k}-n_{k}\right)} \\
& =1+\sum_{k=1}^{\infty} \frac{\left[1+V_{k} n_{k}\right]}{\left(q_{1}-n_{1}\right)\left(q_{2}-n_{2}\right) \ldots\left(q_{k}-n_{k}\right)}
\end{aligned}
$$

Proof. Immediate from (2.4) 'Coverings of parabolic lattice rays' and Lemma (2.5).

(2.7) Lemma (Decreasing covolume). Let $(A, i)$ be a parabolic lattice ray, and let $p:(B, j) \longrightarrow(A, i)$ be a covering. Choose a base-ray in $(B, j)$, say with vertex sequence $b_{0}^{0}, b_{1}^{0}, b_{2}^{0}, \ldots$ If for some $k \geq 1$, we have:

$\left(E_{0}^{B}\left(b_{0}^{k}\right), j\right)=$

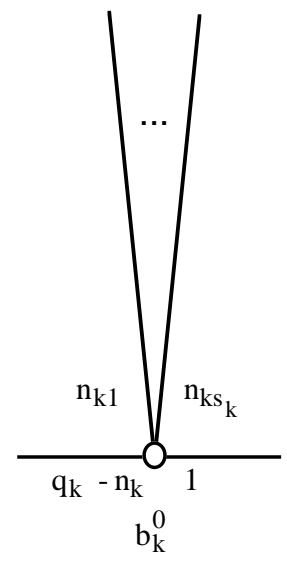

for some $n_{k}$ such that $1<n_{k}<q_{k}$, and for $n_{k 1}, \ldots, n_{k s_{k}}$ satisfying

$$
n_{k 1}+\cdots+n_{k s_{k}}=n_{k}
$$

then:

(i) $\operatorname{Vol}_{b_{0}^{0}}(B, j)$ decreases if we replace $\left(E_{0}^{B}\left(b_{0}^{k}\right), j\right)$ by: 


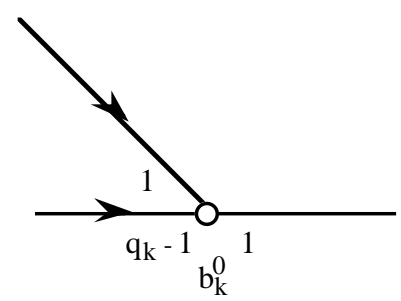

that is, if we replace $n_{k}$ by 1.

(ii) $\operatorname{Vol}_{b_{0}^{0}}(B, j)$ decreases if we replace $\left(E_{0}^{B}\left(b_{0}^{k}\right), j\right)$ by:

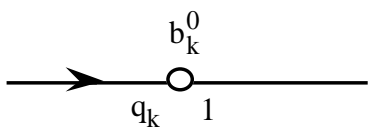

that is, if we replace $n_{k}$ by 0.

Proof. Immediate from Corollary (2.6).

(2.8) Theorem (Canonical reduction of covering). Let $(A, i)$ be a parabolic lattice ray with initial vertex $a_{0}$. Let $p^{\prime}:\left(B^{\prime}, j^{\prime}\right) \longrightarrow(A, i)$ be a covering of $(A, i)$. Then $\left(B^{\prime}, j^{\prime}\right)$ has a canonical 'reduction' $(B, j)$ such that

(i) $(B, j)$ is a covering of $(A, i)$.

(ii) If $\left(B^{\prime}, j^{\prime}\right)$ has infinite fibers then $(B, j)$ has infinite fibers.

(iii) If $b_{0}^{\prime} \in p_{B^{\prime}}^{-1}\left(a_{0}\right)$, then there is a vertex $b_{0} \in p_{B}^{-1}\left(a_{0}\right)$ such that

$$
\operatorname{Vol}_{b_{0}}(B, j) \leq \operatorname{Vol}_{b_{0}^{\prime}}\left(B^{\prime}, j^{\prime}\right)
$$

Thus $(B, j)$ has finite volume if $\left(B^{\prime}, j^{\prime}\right)$ has finite volume.

Proof. The canonical reduction of $\left(B^{\prime}, j^{\prime}\right)$ is defined as follows: Let $a_{k} \in V A$ and suppose $E_{0}^{A}\left(a_{k}\right)=\left\{e_{k a}, e_{k b}\right\}$ where $i\left(e_{k a}\right)=q_{k}$ and $i\left(e_{k b}\right)=1$, that is, $e_{k a}$ points towards $a_{0}$ and $e_{k b}$ points towards the unique end of $(A, i)$. Choose a base-ray in $\left(B^{\prime}, j^{\prime}\right)$ with initial vertex $b_{0}^{\prime}$, and let $\left(f_{1}^{\prime}, f_{2}^{\prime}, \ldots\right)$ be the edge sequence of the base-ray of $\left(B^{\prime}, j^{\prime}\right)$ with $\partial_{0} f_{t}^{\prime}=b_{t-1}^{\prime}, t=1,2, \ldots$

For $k \geq 1$, let $b_{k}^{\prime} \in p^{\prime-1}\left(a_{k}\right)$ be the unique inverse image of $a_{k}$ along the base-ray of $\left(B^{\prime}, j^{\prime}\right)$. Then as in (2.4) ('Coverings of parabolic lattice rays') we have

$$
E_{0}^{B^{\prime}}\left(b_{k}^{\prime}\right):=F_{0}^{B^{\prime}}\left(b_{k}^{\prime}\right) \cup\left\{f_{k a}^{\prime}, f_{k b}^{\prime}\right\}
$$

where $f_{k a}^{\prime}$ and $f_{k b}^{\prime}$ are on the chosen base-ray, $f_{k a}^{\prime}$ points towards the initial vertex of the base-ray and $f_{k b}^{\prime}$ points towards the end, with

$$
j^{\prime}\left(f_{k a}^{\prime}\right)=q_{k}-n_{k}
$$




$$
j^{\prime}\left(f_{k b}^{\prime}\right)=1
$$

and

$$
F_{0}^{B^{\prime}}\left(b_{k}^{\prime}\right):=\left\{f_{k 1}^{\prime}, f_{k 2}^{\prime}, \ldots f_{k s_{k}}^{\prime}\right\}
$$

with

$$
\begin{gathered}
j^{\prime}\left(f_{k 1}^{\prime}\right)=n_{k 1} \\
j^{\prime}\left(f_{k 2}^{\prime}\right)=n_{k 2} \\
\vdots \\
j^{\prime}\left(f_{k s_{k}}^{\prime}\right)=n_{k s_{k}}
\end{gathered}
$$

say, where

$$
n_{k 1}+n_{k 2}+\cdots+n_{k s_{k}}=n_{k} .
$$

We define the canonical reduction $(B, j)$ of $\left(B^{\prime}, j^{\prime}\right)$ as follows. The (edge-indexed) graph $(B, j)$ will be a parabolic edge-indexed tree. Let $\left(f_{1}, f_{2}, \ldots\right)$ be the edge sequence of the base-ray of $(B, j)$. For each $t=1,2, \ldots$ we set

$$
\begin{aligned}
& j\left(f_{t}\right)=1 \\
& j\left(\bar{f}_{t}\right)=q_{t}-1, \quad \text { if } n_{t}>0 \text { in }\left(B^{\prime}, j^{\prime}\right), \\
& q_{t}, \quad \text { if } n_{t}=0 \text { in }\left(B^{\prime}, j^{\prime}\right) \text {. }
\end{aligned}
$$

To each vertex $b_{k}$ on the base-ray of $(B, j)$ such that $\left|E_{0}^{B^{\prime}}\left(b_{k}^{\prime}\right)\right|>2$ in $\left(B^{\prime}, j^{\prime}\right)$ we attach a 'branch' to $b_{k}$ of the form:

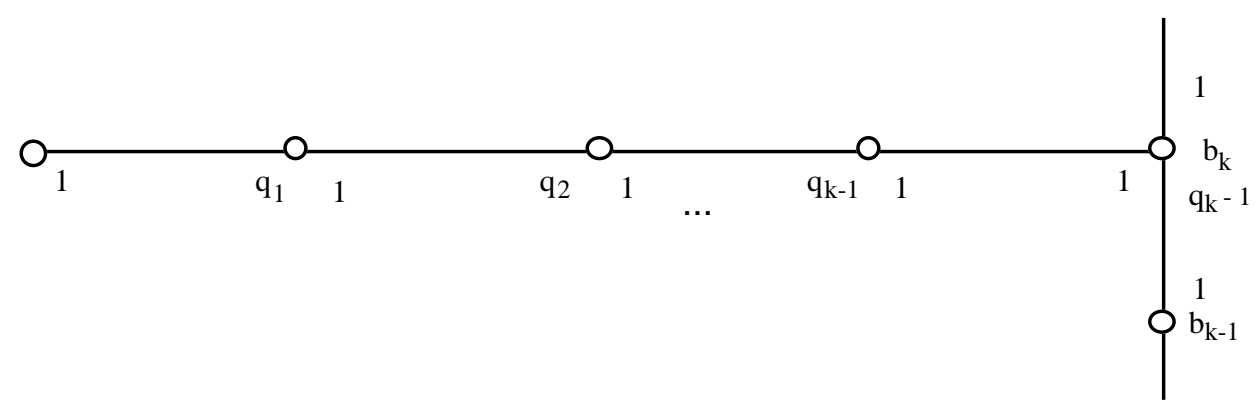

By construction, $(B, j)$ is a covering of $(A, i)$. It is clear that $(B, j)$ has infinite fibers if and only if $\left(B^{\prime}, j^{\prime}\right)$ has infinite fibers. Further, using Lemma $(2.7)(\mathrm{i})$ ('Decreasing covolume'), we can easily check that

$$
\operatorname{Vol}_{b_{0}}(B, j)<\operatorname{Vol}_{b_{0}^{\prime}}\left(B^{\prime}, j^{\prime}\right)
$$


Therefore, if $\left(B^{\prime}, j^{\prime}\right)$ has finite volume, $(B, j)$ also has finite volume.

We will sometimes use the fact that the canonical reduction $(B, j)$ has a distinguished base-ray, and we will refer to this as 'the' base-ray of $(B, j)$.

(2.9) Corollary. If the canonical reduction $(B, j)$ of $\left(B^{\prime}, j^{\prime}\right)$ has infinite volume, then $\left(B^{\prime}, j^{\prime}\right)$ has infinite volume.

(2.10) Remark. We may apply the Bass-Rosenberg volume formula (1.6 (b) and [R]):

$$
\operatorname{Vol}_{b_{0}}(B, j)=\operatorname{Vol}_{a_{0}}(A, i) \times \operatorname{Vol}_{(B, j)}\left(p^{-1}\left(a_{0}\right)\right)
$$

where $a_{0}=p\left(b_{0}\right)$, to compute the volume of the canoncial reduction $(B, j)$.

This yields the following:

(2.11) Lemma. Let $(A, i)$ be a parabolic lattice ray with initial vertex $a_{0}$ :

$(A, i)=$

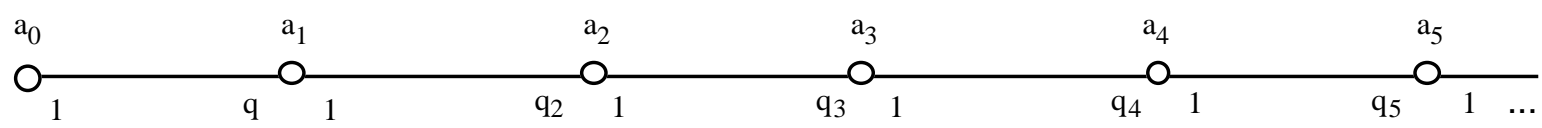

with almost all $q_{k} \geq 2$. Let $p^{\prime}:\left(B^{\prime}, j^{\prime}\right) \rightarrow(A, i)$ be a covering of $(A, i)$. For the canonical reduction $(B, j)$ of $\left(B^{\prime}, j^{\prime}\right)$ we have

$$
\operatorname{Vol}_{(B, j)}\left(p^{-1}\left(a_{0}\right)\right)=1+\sum_{k \text { s.t. } \Delta\left(f_{k}\right)<q_{k}} \frac{q_{1} q_{2} \cdots q_{k-1}}{\Delta\left(f_{1}\right) \Delta\left(f_{2}\right) \cdots \Delta\left(f_{k}\right)},
$$

where $\left(f_{1}, f_{2}, \ldots\right)$ is the edge sequence of the base-ray of $(B, j)$, (hence $1=j\left(f_{1}\right)=$ $\left.j\left(f_{2}\right)=\ldots\right)$, and so $\Delta\left(f_{k}\right)=j\left(\overline{f_{k}}\right) / j\left(f_{k}\right)=j\left(\overline{f_{k}}\right)$. Let $b_{0} \in p_{B}^{-1}\left(a_{0}\right)$ denote the initial vertex of the base-ray of $(B, j)$. We have $\operatorname{Vol}_{b_{0}}(B, j)<\infty$ if and only if

$$
\sum_{k \text { s.t. } \Delta\left(f_{k}\right)<q_{k}} \frac{q_{1} q_{2} \cdots q_{k-1}}{j\left(\overline{f_{1}}\right) j\left(\overline{f_{2}}\right) \cdots j\left(\overline{f_{k}}\right)}<\infty . \square
$$

(2.12) Ray classification theorem. Let $(A, i)$ be a parabolic lattice ray with notation as in Lemma (2.11). Then $(A, i)$ has a covering $p:(B, j) \rightarrow(A, i)$ with infinite fibers and finite volume if and only if the sequence $\left\{q_{k}\right\}_{k=1}^{\infty}$ is unbounded.

Proof. Suppose that the sequence $\left\{q_{k}\right\}_{k=1}^{\infty}$ is bounded. That is, suppose that there exists $N$ such that $q_{k}<N$ for each $k \geq 1$. Asume that we have a covering $p^{\prime}:\left(B^{\prime}, j^{\prime}\right) \rightarrow(A, i)$ such that $\left(B^{\prime}, j^{\prime}\right)$ has infinite fibers. We show that $\left(B^{\prime}, j^{\prime}\right)$ has infinite volume.

Let $(B, j)$ be the canonical reduction of $\left(B^{\prime}, j^{\prime}\right)$ and let $p:(B, j) \rightarrow(A, i)$ be the induced covering of $(A, i)$. Let $b_{0} \in p_{B}^{-1}\left(a_{0}\right)$ be the initial vertex of the base-ray of $(B, j)$. 
By Lemma (2.11), we have $\operatorname{Vol}_{b_{0}}(B, j)<\infty$ if and only if

$$
\begin{aligned}
S: & =\sum_{k \text { s.t. } \Delta\left(f_{k}\right)<q_{k}} \frac{q_{1} q_{2} \ldots q_{k-1}}{\Delta\left(f_{1}\right) \Delta\left(f_{2}\right) \ldots \Delta\left(f_{k}\right)} \\
& =\sum_{k \text { s.t. } \Delta\left(f_{k}\right)<q_{k}} \frac{q_{1}}{\Delta\left(f_{1}\right)} \frac{q_{2}}{\Delta\left(f_{2}\right)} \ldots \frac{q_{k-1}}{\Delta\left(f_{k-1}\right)} \frac{1}{\Delta\left(f_{k}\right)} \\
& <\infty .
\end{aligned}
$$

Since $\Delta\left(f_{k}\right) \leq q_{k}<N$ for each $k \geq 1$, we have

$$
S>\frac{1}{N}\left(\sum_{k \text { s.t. } \Delta\left(f_{k}\right)<q_{k}} \frac{q_{1}}{\Delta\left(f_{1}\right)} \frac{q_{2}}{\Delta\left(f_{2}\right)} \cdots \frac{q_{k-1}}{\Delta\left(f_{k-1}\right)}\right),
$$

but $\Delta\left(f_{k}\right) \leq q_{k}$ for each $k \geq 1$, so

$$
\frac{q_{k}}{\Delta\left(f_{k}\right)} \geq 1
$$

Hence

$$
S \quad>\quad \frac{1}{N} \sum_{k=1}^{\infty} 1=\infty .
$$

Conversely, suppose that $(A, i)$ has unbounded index. We construct a covering $p$ : $(B, j) \rightarrow(A, i)$ with infinite fibers and finite volume.

We choose the least $k$ such that $q_{k}>1$. For $l=0, \ldots k$ we define $b_{0}, \ldots, b_{k}$ to be vertices of $B$, with $p\left(b_{l}\right)=a_{l}$, and for $l=0, \ldots, k-1$, we define:

$$
\left(E_{0}^{B}\left(b_{l}\right), j\right):=\left(E_{0}^{A}\left(a_{l}\right), i\right)
$$

We define $\left(E_{0}^{B}\left(b_{k}\right), j\right)$ to be:

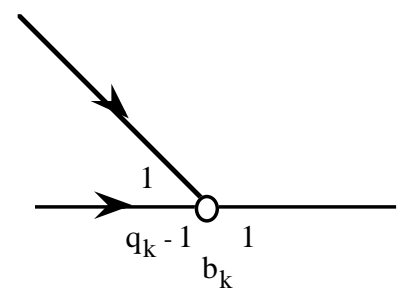

The vertices $b_{0}, \ldots, b_{k}$ become part of the base-ray of $(B, j)$. To construct the desired covering $(B, j)$ of $(A, i)$, we imitate the construction of the canonical reduction of a covering of $(A, i)$. We construct a branch denoted $B_{k}$ from the vertex $b_{k}$ on the base-ray of the form: 
$(2)$

$B_{k}:=$

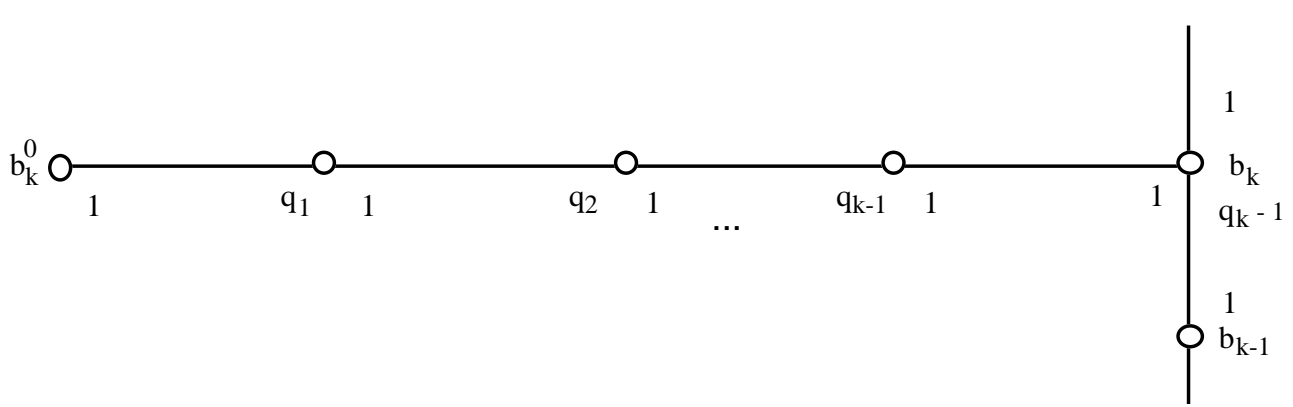

Let $b_{k}^{0}$ be the terminal vertex of the branch $B_{k}$, other than $b_{0}$. Then $b_{k}^{0} \in p_{B}^{-1}\left(a_{0}\right)$. By Lemma (2.11), we have:

$$
V_{k}:=\operatorname{Vol}_{(B, j)}\left(b_{k}^{0}\right)=\frac{q_{1} q_{2} \ldots q_{k-1}}{\Delta\left(f_{1}\right) \Delta\left(f_{2}\right) \ldots \Delta\left(f_{k}\right)} .
$$

\section{(2.13) Branch vertices.}

Next we choose $a_{k+n_{0}} \in V A$ such that

$$
q_{k+n_{0}}-1>2 q_{k},
$$

which is possible since the sequence $\left\{q_{k}\right\}$ is unbounded. We define $b_{k+1}, \ldots, b_{k+n_{0}}$ to be vertices of $B$, with $p\left(b_{k+l}\right)=a_{k+l}, l=1, \ldots, n_{0}$. For $l=1, \ldots, n_{0}-1$, we choose

$$
\left(E_{0}^{B}\left(b_{k+l}\right), j\right):=\left(E_{0}^{A}\left(a_{k+l)}, i\right) .\right.
$$

We choose $\left(E_{0}^{B}\left(b_{k+n_{0}}\right), j\right)$ as follows:

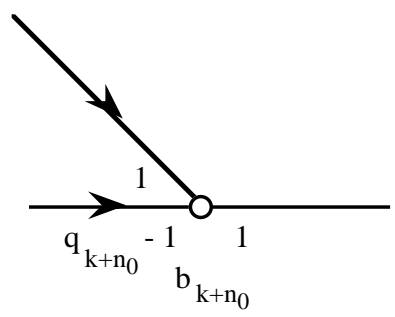


The vertices $b_{k+1}, \ldots, b_{k+n_{0}}$ also become part of the base-ray of $(B, j)$. We construct a branch denoted $B_{k+n_{0}}$ from $b_{k+n_{0}}$ of the form:

$B_{k+n_{0}}:=$

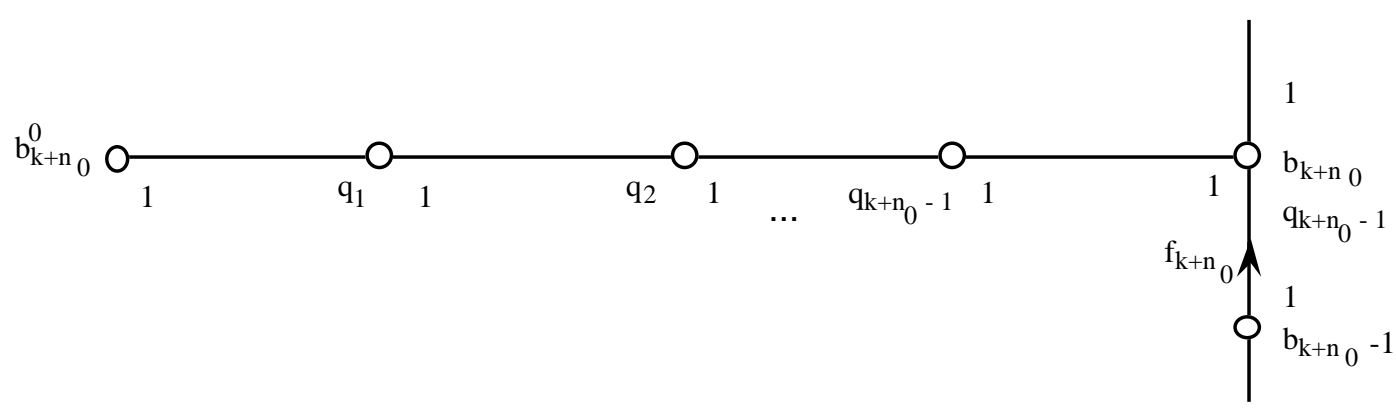

Let $b_{k+n_{0}}^{0}$ be the terminal vertex of the branch $B_{k+n_{0}}$, other than $b_{0}$. Then $b_{k+n_{0}}^{0} \in p_{B}^{-1}\left(a_{0}\right)$. Let $\left(f_{1}, f_{2}, \ldots, f_{k+n_{0}}\right)$ be the edge sequence of the base-ray constructed so far. By Lemma (2.11), we have:

$$
\begin{aligned}
V_{k+n_{0}}:=\operatorname{Vol}_{(B, j)}\left(b_{k+n_{0}}^{0}\right) & =\frac{q_{1} q_{2} \ldots q_{k+n_{0}-1}}{\Delta\left(f_{1}\right) \Delta\left(f_{2}\right) \ldots \Delta\left(f_{k+n_{0}}\right)} \\
& =\frac{q_{1} q_{2} \ldots q_{k-1}}{\Delta\left(f_{1}\right) \Delta\left(f_{2}\right) \ldots \Delta\left(f_{k}\right)} q_{k} \frac{q_{k+1} \ldots q_{k+n_{0}-1}}{\Delta\left(f_{k+1}\right) \ldots \Delta\left(f_{k+n_{0}-1}\right)} \frac{1}{\Delta f\left(k+n_{0}\right)} \\
& =V_{k} q_{k} \frac{q_{k+1}}{q_{k+1}} \ldots \frac{q_{k+n_{0}-1}}{q_{k+n_{0}-1}} \frac{1}{q_{k+n_{0}-1}} \\
& =V_{k} \frac{q_{k}}{q_{k+n_{0}}-1} \\
& <V_{k} \frac{q_{k}}{2 q_{k}} \\
& =\frac{V_{k}}{2} .
\end{aligned}
$$

We iterate the definition of $(B, j)$ in this way, repeating the construction from $(2.13)$, for each $l=1,2, \ldots$, choosing $b_{k+n_{l}}$ for $n_{l}>n_{l-1}$, such that

$$
q_{k+n_{l}}-1>2 q_{k+n_{l-1}} .
$$


(This is possible, as the sequence $\left\{q_{k}\right\}$ is unbounded). We choose vertices $b_{k+n_{l-1}}, \ldots, b_{k+n_{l}}$ which will become part of the base-ray of $(B, j)$, with $p\left(b_{k+n_{l-1}}\right)=a_{k+n_{l-1}}, \ldots, p\left(b_{k+n_{l}}\right)=$ $a_{k+n_{l}}$, and we choose

$$
\begin{gathered}
\left(E_{0}^{B}\left(b_{k+n_{l-1}}\right), j\right):=\left(E_{0}^{A}\left(a_{k+n_{l-1}}\right), i\right) \\
\vdots \\
\left(E_{0}^{B}\left(b_{k+n_{l}-1}\right), j\right):=\left(E_{0}^{A}\left(a_{k+n_{l}-1}\right), i\right) .
\end{gathered}
$$

We choose $\left(E_{0}^{B}\left(b_{k+n_{l}}\right), j\right)$ as follows:

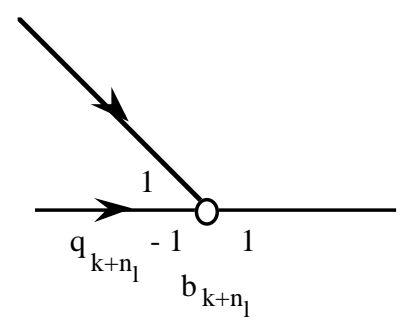

For each $l=1,2, \ldots$, we construct a branch denoted $B_{k+n_{l}}$ from $b_{k+n_{l}}$ with terminal vertex $b_{k+n_{l}}^{0}$ other than $b_{0}$ :

$B_{k+n_{l}}:=$

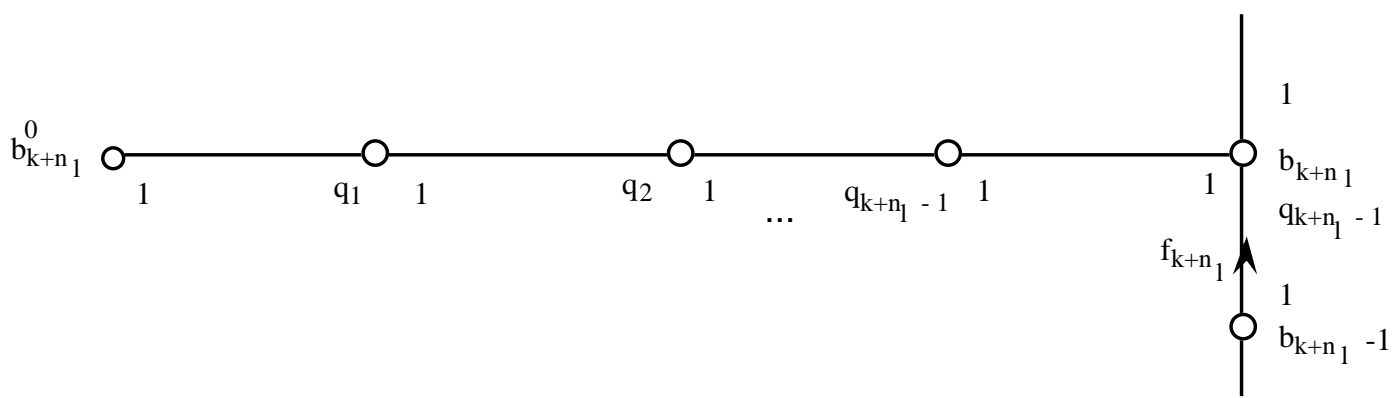

For each $l=1,2, \ldots$ we have: 


$$
\begin{aligned}
V_{k+n_{l}}: & =\operatorname{Vol}_{(B, j)}\left(b_{k+n_{l}}^{0}\right) \\
& =\frac{q_{1} q_{2} \ldots q_{k+n_{l}-1}}{\Delta\left(f_{1}\right) \Delta\left(f_{2}\right) \ldots \Delta\left(f_{k+n_{l}}\right)} \\
& \stackrel{(14)}{=} V_{k}\left(\frac{q_{k}}{q_{k+n_{0}}-1}\right)\left(\frac{q_{k+n_{0}}}{q_{k+n_{1}}-1}\right)\left(\frac{q_{k+n_{1}}}{q_{k+n_{2}}-1}\right) \ldots\left(\frac{q_{k+n_{(l-1)}}}{q_{k+n_{l}}-1}\right)
\end{aligned}
$$

since we branch only at vertices $b_{k+n_{0}}, b_{k+n_{1}}, \ldots$

$$
\begin{aligned}
& <V_{k}\left(\frac{q_{k}}{2 q_{k}}\right)\left(\frac{q_{k+n_{0}}}{2 q_{k+n_{0}}}\right)\left(\frac{q_{k+n_{1}}}{2 q_{k+n_{1}}}\right) \ldots\left(\frac{q_{k+n_{(l-1)}}}{2 q_{k+n_{(l-1)}}}\right) \\
& =\frac{V_{k}}{2^{l+1}} .
\end{aligned}
$$

It follows that:

$$
\begin{aligned}
\operatorname{Vol}_{(B, j)}\left(p^{-1}\left(a_{0}\right)\right) & :=\operatorname{Vol}_{(B, j)}\left(b_{k}^{0}\right)+\operatorname{Vol}_{(B, j)}\left(b_{k+n_{0}}^{0}\right)+\operatorname{Vol}_{(B, j)}\left(b_{k+n_{1}}^{0}\right)+\ldots \\
& <V_{k}+\frac{V_{k}}{2}+\frac{V_{k}}{2^{2}}+\ldots \\
& <\infty
\end{aligned}
$$

By construction, $(B, j)$ is a covering of $(A, i)$, and $(B, j)$ has infinite fibers, since the sequence $\left\{q_{k}\right\}$ is unbounded. $\square$

\section{Parabolic lattice trees}

In this section we prove Theorem (0.2) in the case that $(A, i)$ is a parabolic lattice tree. We seek a covering $p:(B, j) \longrightarrow(A, i)$ with infinite fibers and finite volume. This gives rise to a non-uniform $G$-lattice, where $G=A u t(X), X=\widetilde{(A, i)}$.

Fix a terminal vertex $a_{0} \in V A$ and let $\left(A_{0}, i\right)=\left(a_{0}, a_{1}, a_{2}, \ldots\right)$ be the (vertex sequence of the) path from $a_{0}$ to the end $\epsilon_{A}$. Then we may view $(A, i)$ as a 'decoration' of the chosen base-ray $\left(A_{0}, i\right)$. That is, $(A, i)=$

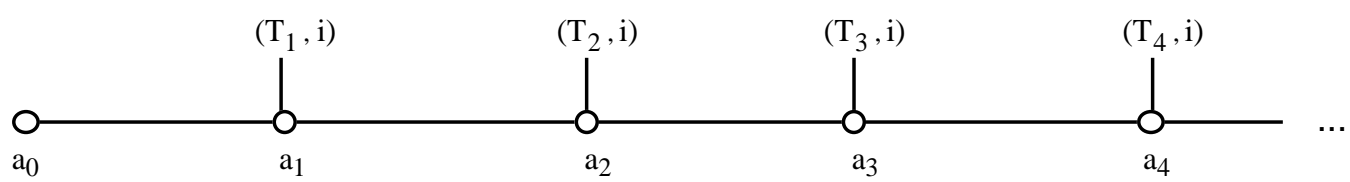

where $\left(T_{k}, i\right)$ is a finite (possibly empty) dominant-rooted edge-indexed subtree attatched to $a_{k} \in V A_{0}$.

(3.1) Lemma. Let $(A, i)$ be a parabolic lattice tree and $\left(A_{0}, i\right)$ a chosen base ray of $(A, i)$. Let $p_{0}:\left(B_{0}, j\right) \longrightarrow\left(A_{0}, i\right)$ be a covering and let $(B, j)$ be obtained by attaching the finite dominant-rooted edge-indexed subtree $\left(T_{k}, i\right)$ (specified above) to every vertex in $p_{B_{0}}^{-1}\left(a_{k}\right)$. Then $p:(B, j) \longrightarrow(A, i)$ is a covering.

Proof. Obvious. 
Let $(A, i)$ be a parabolic lattice tree. Choose $\left(A_{0}, i\right)=\left(e_{1}, e_{2}, e_{3}, \ldots\right)$ to be a base-ray of $(A, i)$. By Theorem (2.12),

\section{$\left(A_{0}, i\right)$ has a covering with infinite fibers and finite volume if and only if the sequence $\left\{q_{k}=i\left(\overline{e_{k}}\right)\right\}_{k=0}^{\infty}$ is unbounded.}

\section{(3.2) Decorated cover of a base-ray of $(A, i)$.}

We assume that $(A, i)$ has a base-ray with the sequence $\left\{q_{k}=i\left(\overline{e_{k}}\right)\right\}_{k=0}^{\infty}$ unbounded, and let $p^{\prime}:\left(B^{\prime}, j^{\prime}\right) \longrightarrow\left(A_{0}, i\right)$ be a covering of the base-ray $\left(A_{0}, i\right)$ with infinite fibers and finite volume. We construct the canonical reduction $\left(B_{0}, j\right)$ of $\left(B^{\prime}, j^{\prime}\right)$ as in Theorem (2.8). Then $\left(B_{0}, j\right)$ has infinite fibers and finite volume. At every vertex $a_{k}$ in $V A_{0}$ is attached a (finite, possibly empty) dominant-rooted edge-indexed subtree, called $\left(T_{k}, i\right)$. We now construct a covering of $(A, i)$ by 'decorating' $\left(B_{0}, j\right)$. We attach a copy of $\left(T_{k}, i\right)$ to each $b_{k}$ in $p^{-1}\left(a_{k}\right)$, and call the resulting edge-indexed graph $(B, j)$. The following theorem shows that this gives the desired covering of $(A, i)$.

(3.3) Theorem. Let $(A, i)$ be a parabolic lattice tree. Fix a terminal vertex $a_{0}$ of $(A, i)$ such that the path $\left(e_{1}, e_{2}, \ldots\right)$ from $a_{0}$ to the (unique) end of $(A, i)$ has unbounded index. Then there is a covering $p:(B, j) \longrightarrow(A, i)$ with infinite fibers and finite volume.

\section{(3.4) Remark.}

The following example shows that a parabolic lattice tree may have unbounded index, however, every path directed towards the end of the edge-indexed quotient has bounded index. $(A, i)=$

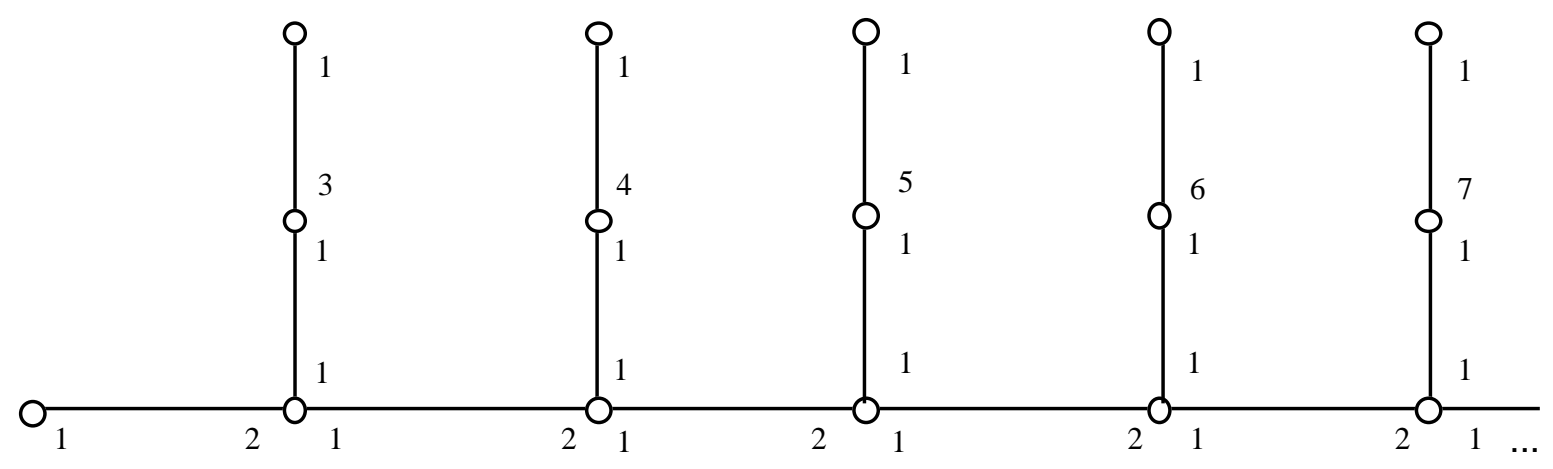

Proof of (3.3). Let $\left(A_{0}, i\right)=\left(e_{1}, e_{2}, \ldots\right)$ be a base-ray of $(A, i)$ and let $p_{0}:\left(B_{0}, j\right) \longrightarrow$ $\left(A_{0}, i\right)$ be the covering of $\left(A_{0}, i\right)$ with infinite fibers and finite volume as in (3.2). Let $p:(B, j) \longrightarrow(A, i)$ be the decorated covering of $\left(B_{0}, j\right)$ as in $(3.2)$. Let $b_{0} \in p^{-1}\left(a_{0}\right)$ be the distinguished initial vertex of the base-ray of $\left(B_{0}, j\right)$.

By Lemma $(3.1)$, we readily see that $(B, j)$ is a covering of $(A, i)$, since $\left(B_{0}, j\right)$ is a covering of $\left(A_{0}, i\right)$. Clearly

$(B, j)$ has infinite fibers $\Longleftrightarrow\left(B_{0}, j\right)$ has infinite fibers. 
Using the Bass-Rosenberg Volume Formula $([\mathrm{R}])$, we compute the volume of $(B, j)$ :

$$
\operatorname{Vol}_{\left(b_{0}\right)}(B, j)=\operatorname{Vol}_{\left(a_{0}\right)}(A, i) \times \operatorname{Vol}_{(B, j)} p^{-1}\left(a_{0}\right) .
$$

The volume at a single vertex $b$ in the fiber $p^{-1}\left(a_{0}\right)$ depends only on the indices along the unique path from $b_{0}$ to $b$, and these indices are unchanged by decorating $\left(B_{0}, j\right)$. Hence it follows that

$$
\operatorname{Vol}_{\left(B_{0}, j\right)} p^{-1}\left(a_{0}\right)=\operatorname{Vol}_{(B, j)} p^{-1}\left(a_{0}\right)
$$

Since $\left(B_{0}, j\right)$ has finite volume, so does $(B, j)$.

\section{(3.5) Remark.}

Let $(A, i)$ be a parabolic lattice tree. The fact that $(A, i)$ has a unique end implies that

$$
\text { some ray of }(A, i) \text { has bounded index }
$$

\section{if and only if}

every ray of $(A, i)$ has bounded index.

(3.6) Lemma. Let $p:(B, j) \longrightarrow(A, i)$ be a covering of edge-indexed trees, and let $b_{0} \in(B, j)$. Then if $p^{-1}\left(a_{0}\right)=b_{0}$, we have $\operatorname{Vol}_{b_{0}}(B, j)=\operatorname{Vol}_{a_{0}}(A, i)$.

This lemma is a special case of the Bass-Rosenberg volume formula and was discovered independently in $[\mathrm{CE}]$ in the case that $(A, i)$ and $(B, j)$ are edge-indexed trees.

(3.7) Theorem. Let $(A, i)$ be a parabolic lattice tree. If every path towards the end of $(A, i)$ has bounded index, then $(A, i)$ has no covering with infinite fibers and finite volume.

Proof. Fix a terminal vertex $a_{0}$ of $(A, i)$ and let $\left(a_{1}, a_{2}, \ldots\right)$ be the vertex sequence from $a_{0}$ to the unique end of $(A, i)$. Assume that there is a covering $p:(B, j) \longrightarrow(A, i)$ with infinite fibers and finite volume. Traversing the path $\gamma=\left(a_{1}, a_{2}, \ldots\right)$, we consider the preimages $p^{-1}\left(a_{1}\right), p^{-1}\left(a_{2}\right), \ldots$ for $r \geq 1$, and $l \geq 1$. Let $b_{r}^{l} \in p^{-1}\left(a_{r}\right)$. Let $T_{r}$ be a finite (possibly empty) subtree attached to $a_{r}$. We have

$$
\operatorname{deg}\left(a_{r}\right) \quad:=q_{r-1}+1+s_{r}
$$

where $q_{r-1}$ is the index of the incoming edge to $a_{r}$ along the chosen base-ray and $s_{r}$ is the sum of the indices of edges in $T_{r}$ emanating from $a_{r}$. Then $p_{b_{r}^{l}}^{-1}\left(a_{r}\right)$ looks like 


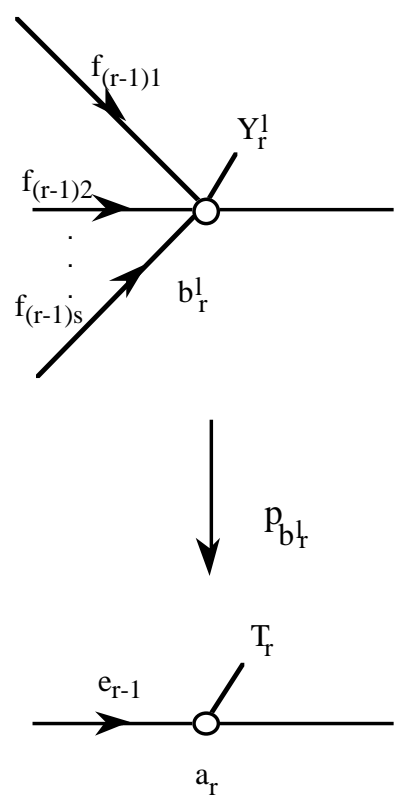

where

$$
j\left(\bar{f}_{(r-1) 1}\right)+\cdots+j\left(\bar{f}_{(r-1) s}\right)=i\left(\bar{e}_{r-1}\right)=q_{r-1},
$$

and $Y_{r}^{l}$ is a covering of $T_{r}$.

We replace $Y_{r}^{l}$ by a copy of $T_{r}$, called $T_{r}^{l}$, and by Lemma (3.6) (and the fact that the trees $Y_{r}^{l}$ and $T_{r}$ are dominant rooted), this does not change the volume of $(B, j)$. We 'prune' the finite subtrees $T_{r}^{l}$, for $r \geq 1$, from $(B, j)$ and call the resulting edge-indexed graph $\left(B_{0}, j_{0}\right)$. We claim that there is a covering $p_{0}:\left(B_{0}, j\right) \longrightarrow\left(A_{0}, i\right)$. For $a_{r} \in V A_{0}$, we have the local picture:

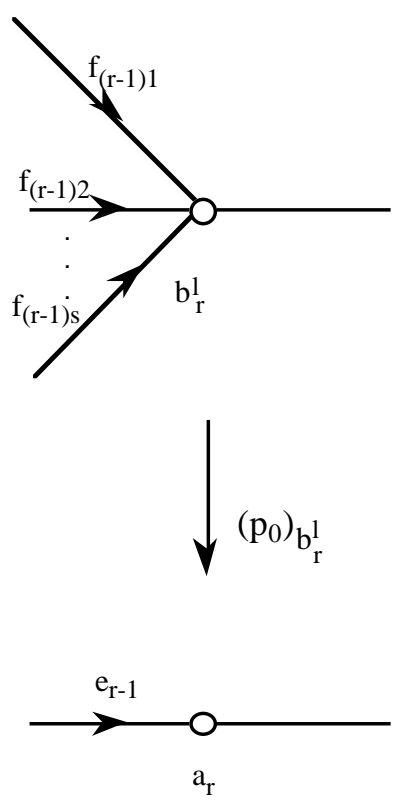


where

$$
j\left(\bar{f}_{(r-1) 1}\right)+\cdots+j\left(\bar{f}_{(r-1) s}\right) \quad=i\left(\bar{e}_{r-1}\right)=q_{r-1},
$$

and hence $p_{0}$ is a covering.

Repeating this for every $l=2,3, \ldots$ and for each $r=2,3, \ldots$ we obtain a covering $\left(B^{\prime}, j^{\prime}\right)$ of the base-ray $\left(A_{0}, i\right)$ to which finite trees $T_{r}$ are attached to each $a_{r} \in V A_{0}$. Moreover

$\left(B^{\prime}, j^{\prime}\right)$ has infinite fibers and finite volume

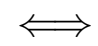

$(B, j)$ has infinite fibers and finite volume.

However, $\left(A_{0}, i\right)$ has bounded index by hypothesis, so by Theorem $(2.12)$, the coverings $\left(B^{\prime}, j^{\prime}\right)$ and $\left(B_{0}, j\right)$ cannot exist. Hence there can be no covering $p:(B, j) \longrightarrow(A, i)$ with infinite fibers and finite volume.

(3.8) Corollary. In the notation of Theorem (3.7) and its proof we see that a covering $p:(B, j) \longrightarrow(A, i)$ with infinite fibers and finite volume induces a decoration $\left(B^{\prime}, j^{\prime}\right)$ of a covering $p_{0}:\left(B_{0}, j\right) \longrightarrow\left(A_{0}, i\right)$ with infinite fibers and finite volume of the base-ray $\left(A_{0}, i\right)$.

As a corollary to Theorems (3.3) and (3.7) we obtain:

(3.9) Theorem. Let $(A, i)$ be a parabolic lattice tree. Then $(A, i)$ has a covering $p:(B, j) \longrightarrow(A, i)$ with infinite fibers and finite volume if and only if $(A, i)$ contains a ray with unbounded index. $\square$

In private communication, G. Rosenberg observed the following:

(3.10) Lemma (G. Rosenberg). Let $(A, i)$ be a parabolic edge-indexed tree with unique end $\epsilon$. Let $\left(e_{1}, e_{2}, \ldots\right)$ be an infinite path towards $\epsilon$. Set $a_{1}=\partial_{0} e_{1}$ and $q_{k}=i\left(\bar{e}_{k}\right)$. Note that $i\left(e_{k}\right)=1$. Let $p:(B, j) \longrightarrow(A, i)$ be a covering of edge-indexed graphs (thus $(B, j)$ is a parabolic edge-indexed tree with unique end $\widetilde{\epsilon})$. Let $\left(f_{1}, f_{2}, \ldots\right)$ be a lifting of $\left(e_{1}, e_{2}, \ldots\right)$ and set $b_{0}=\partial_{0} f_{1}\left(\right.$ so $\left.p\left(b_{0}\right)=a_{0}\right)$ and $\widetilde{q}_{k}=j\left(\widetilde{f}_{k}\right)$ (again, $\left.j\left(f_{k}\right)=1\right)$. Then we have

$$
\operatorname{Vol}_{b_{0}}(B, j)=\left(\prod_{k=1}^{\infty} \frac{q_{k}}{\widetilde{q}_{k}}\right) \operatorname{Vol}_{a_{0}}(A, i) .
$$

(cf. Corollary (2.6) and Lemma (2.11). This is a generalization of Lemma (2.11) to parabolic lattice trees).

Proof. : Since $A$ is a tree, every edge is separating. For each $n \in \mathbb{Z}_{\geq 0}$, let $\left(A_{n}^{0}, i\right)$ and $\left(A_{n}^{1}, i\right)$ be the two connected components obtained by removing the separating edge $e_{n+1}$, where we assume that $\left(A_{n}^{1}, i\right)$ is the connected component that contains the end of $(A, i)$. Let $a_{n}=\partial_{0} e_{n+1}$. Then $\left(A_{n}^{0}, i, a_{n}\right)$ is a (finite) dominant rooted edge-indexed tree. Similarly, for each $n \in \mathbb{Z}_{\geq 0}$, let $\left(B_{n}^{0}, j\right)$ and $\left(B_{n}^{1}, j\right)$ be the two connected components obtained by removing the separating edge $f_{n+1}$ in $(B, j)$, where we assume that $\left(B_{n}^{1}, j\right)$ is the connected component that contains the end of $(B, j)$. Let $b_{n}=\partial_{0} f_{n+1}$. Then 
$\left(B_{n}^{0}, j, b_{n}\right)$ is also a (finite) dominant rooted edge-indexed tree, and for each $n \in \mathbb{Z}_{\geq 0}$, $p_{n}=\left.p\right|_{\left(B_{n}^{0}, j\right)}:\left(B_{n}^{0}, j\right) \longrightarrow\left(A_{n}^{0}, i\right)$ is a covering of edge-indexed graphs with $p^{-1}\left(a_{n}\right)=\bar{b}_{n}$.

Hence by the Bass-Rosenberg volume formula, we have:

$$
\operatorname{Vol}_{b_{n}}\left(B_{n}^{0}, j\right)=\operatorname{Vol}_{a_{n}}\left(A_{n}^{0}, i\right) .
$$

The change of base-point formula $([\mathrm{BL}],(2.6))$ gives:

$$
\begin{aligned}
\operatorname{Vol}_{b_{n}}\left(B_{n}^{0}, j\right) & =\left(\prod_{k=1}^{n} \widetilde{q}_{k}\right) \operatorname{Vol}_{b_{0}}\left(B_{n}^{0}, j\right) \\
& =\operatorname{Vol}_{a_{n}}\left(A_{n}^{0}, i\right) \\
& =\left(\prod_{k=1}^{n} q_{k}\right) \operatorname{Vol}_{a_{0}}\left(A_{n}^{0}, i\right) .
\end{aligned}
$$

So

$$
\operatorname{Vol}_{b_{0}}\left(B_{n}^{0}, j\right)=\left(\prod_{k=1}^{n} \frac{q_{k}}{\widetilde{q}_{k}}\right) \operatorname{Vol}_{a_{0}}\left(A_{n}^{0}, i\right) .
$$

Taking the limit as $n \longrightarrow \infty$, we get:

$$
\operatorname{Vol}_{b_{0}}(B, j)=\left(\prod_{k=1}^{\infty} \frac{q_{k}}{\widetilde{q}_{k}}\right) \operatorname{Vol}_{a_{0}}(A, i) . \square
$$

\section{(3.11) Remarks.}

(1) $\widetilde{q}_{k} \leq q_{k}$.

(2) If $p$ has infinite fibers, then we must have $\widetilde{q}_{k}<q_{k}$ for infinitely many $k$.

(3) Although the $\widetilde{q}_{k}$ depend on the choice of lifting, the finiteness of the infinite product does not. Also, the $q_{k}$ are bounded (not necessarily uniformly) for some ray in $(A, i)$ if and only if the $q_{k}$ are bounded for every ray in $(A, i)$.

At the time of publication, G. Rosenberg observed that the lemma can be applied to give an alternate proof of Theorems (2.12) and (3.3). We give Rosenberg's proof of Theorem (3.3) below (Theorem (3.12)).

Let $(A, i)$ be a parabolic edge-indexed tree. We say that $(A, i)$ has the bounded ray condition if for some, hence every, ray $\left(e_{1}, e_{2}, \ldots\right)$ towards the unique end of $(A, i)$, there exists an $N>0$ (which may depend on the chosen ray) such that $i\left(\bar{e}_{n}\right)<N$ for all $n=1,2, \ldots$.

(3.12) Theorem (G. Rosenberg). Let $(A, i)$ be a parabolic tree with finite volume. If $(A, i)$ does not satisfy the bounded ray condition, then there is a covering $p:(B, j) \longrightarrow$ $(A, i)$ with infinite fibers and finite volume.

Proof. Let $\left(e_{1}, e_{2}, \ldots\right)$ be a ray of $(A, i)$. Let $q_{k}=i\left(\bar{e}_{k}\right)$. Since $\left\{q_{k}\right\}$ is unbounded, there exists a subsequence $\left\{q_{k_{n}}\right\}$ which grows exponentially, that is, $q_{k_{n+1}}>c q_{k_{n}}$ for some $c>0$. 
We fan $(A, i)$ at each $e_{k_{n}}$ to create a cover $(B, j)$ which has a lifting $\left(f_{1}, f_{2}, \ldots\right)$ of $\left(e_{1}, e_{2}, \ldots\right)$ with $j\left(\bar{f}_{k_{n}}\right)=i\left(\bar{e}_{k_{n}}\right)-1$ for $k_{n}$ in our subsequence. (This process is described in detail in the proof of Theorem (2.12) for parabolic rays, and Theorem (3.3) for parabolic trees.) Then $(B, j)$ has infinite fibers. Moreover for basepoints $a_{0} \in V A$ and $b_{0} \in V B$ :

$$
\begin{array}{r}
\operatorname{Vol}_{b_{0}}(B, j)=\left(\prod_{k=1}^{\infty} \frac{q_{k}}{\widetilde{q}_{k}}\right) \operatorname{Vol}_{a_{0}}(A, i) \\
=\prod_{k=1}^{\infty}\left(1+\frac{1}{\left(q_{k_{n}}-1\right)}\right),
\end{array}
$$

which converges since $\left\{q_{k_{n}}\right\}$ (and hence $\left\{q_{k_{n}}-1\right\}$ ) grows exponentially.

\section{Towers of coverings with infinite fibers and finite volume}

Let $(A, i)$ be the following parabolic lattice ray:

$(A, i)=$

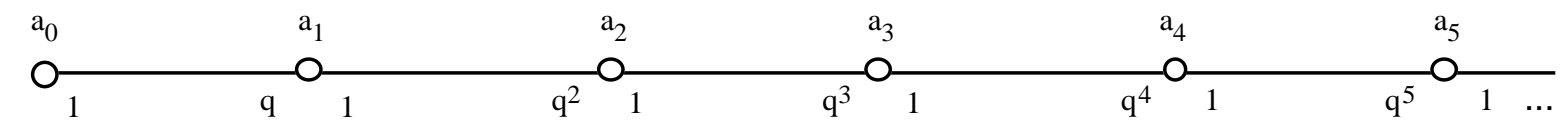

with $q \geq 2$. Since $(A, i)$ has unbounded index, we may use the technique of Theorem $(2.12)$ to construct a covering $p:(B, j) \longrightarrow(A, i)$ such that $(B, j)$ as infinite fibers and finite volume. For $q \geq 3$, the resulting covering looks like: 
$(B, j)=$

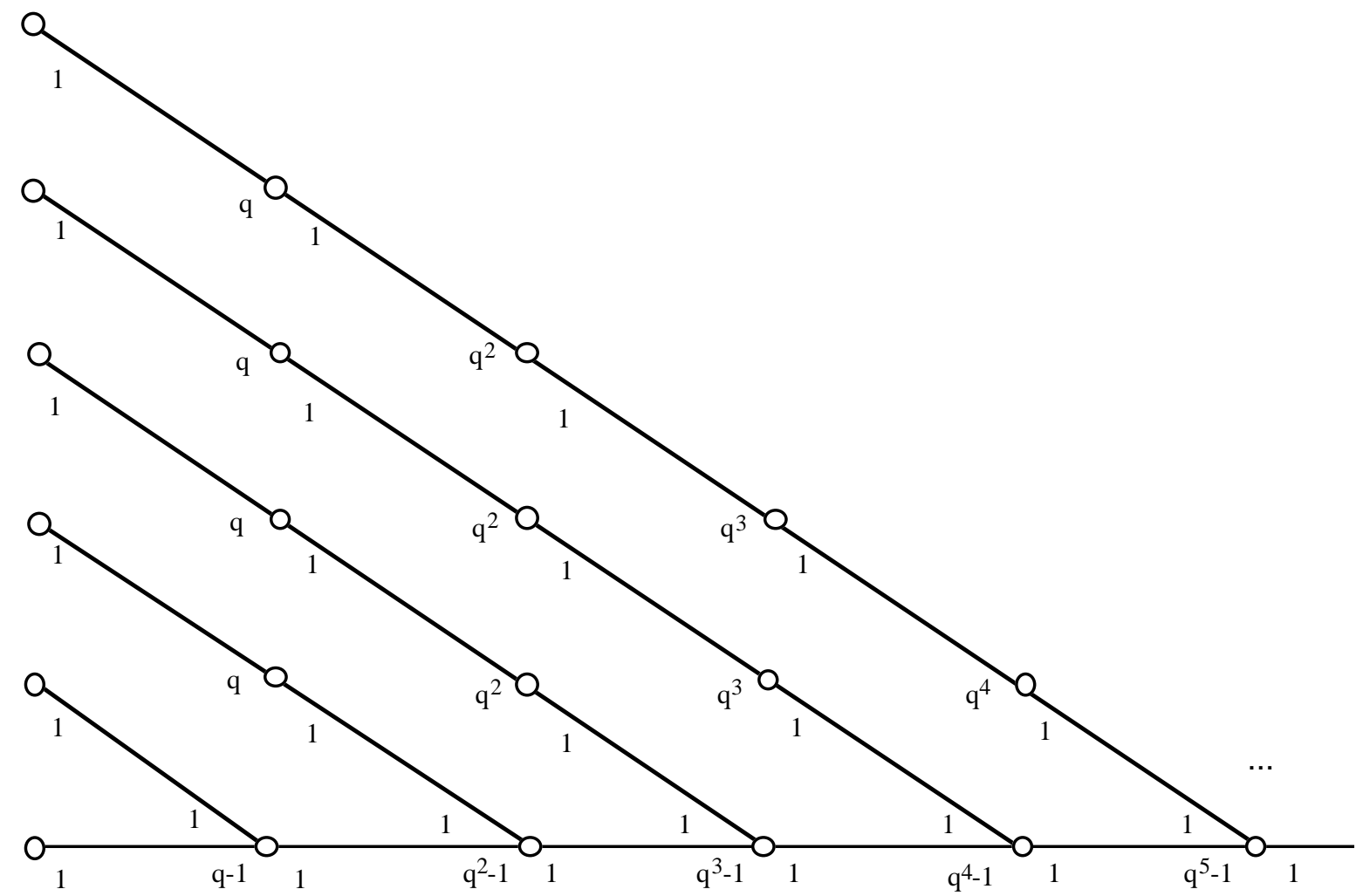

We set $\left(B_{0}, j_{0}\right)=(B, j)$, and we use Lemma (2.7)(ii) ('Decreasing covolume') to modify $\left(B_{0}, j_{0}\right)$ to obtain coverings $\left(B_{l}, j_{l}\right)$ for $l=1,2, \ldots$ of $(A, i)$ with infinite fibers and finite covolume, but smaller covolume than $\left(B_{0}, j\right)$. We set 
$\left(B_{1}, j_{1}\right)=$

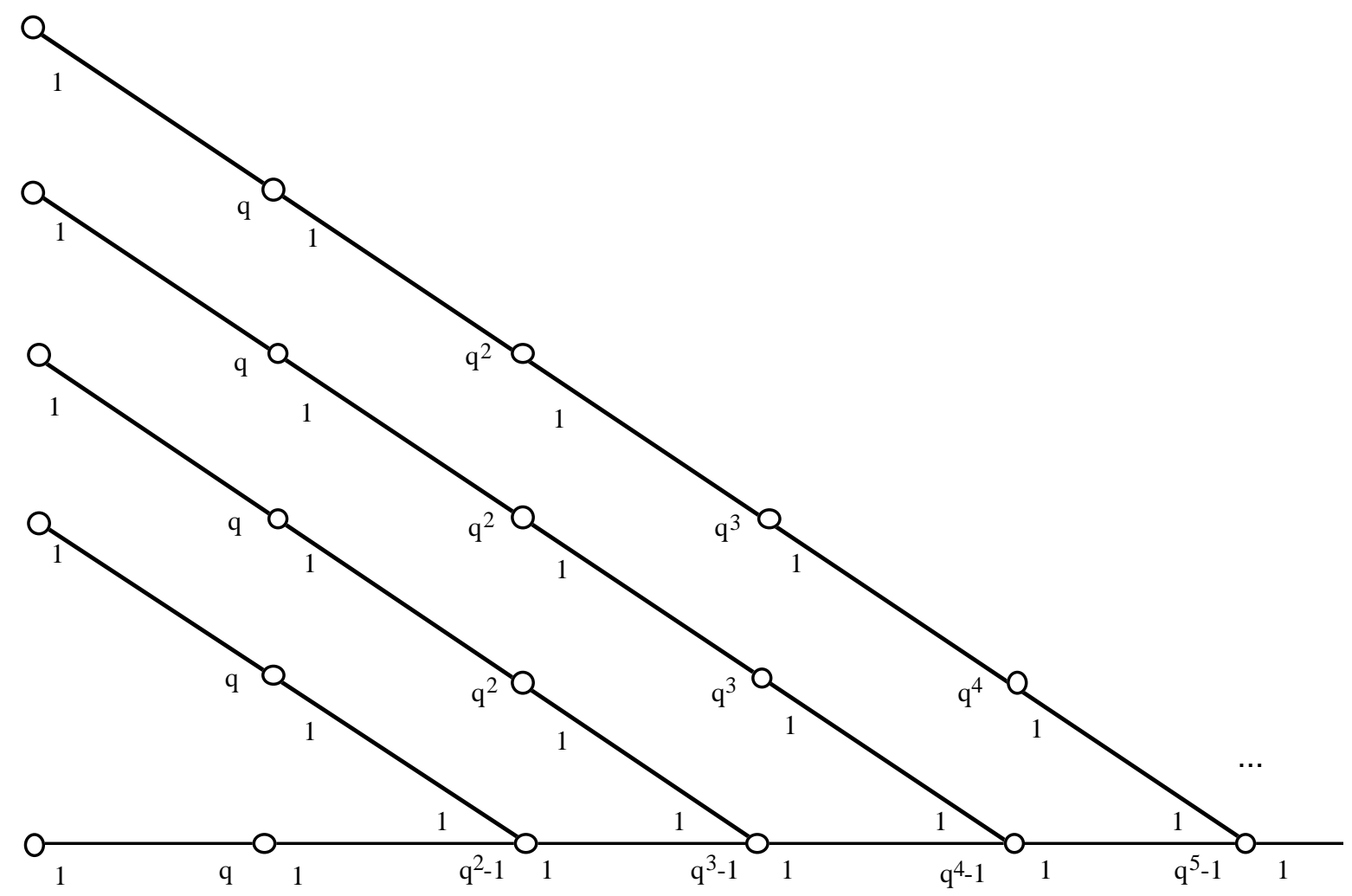


and $\left(B_{2}, j_{2}\right)=$

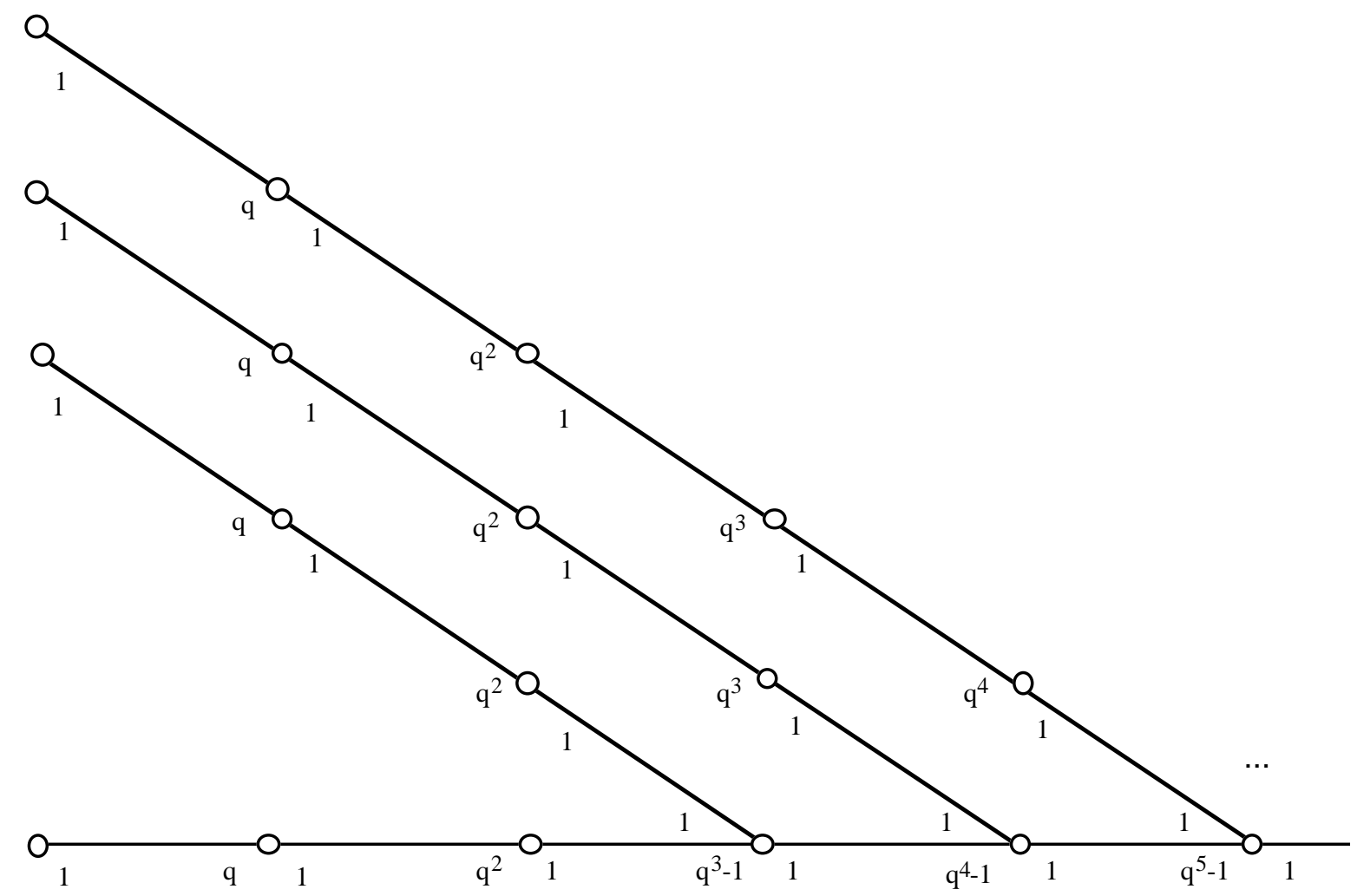

and so on. In this way, we obtain an infinite sequence of coverings:

$$
\left(B_{0}, j_{0}\right) \longrightarrow\left(B_{1}, j_{1}\right) \longrightarrow\left(B_{2}, j_{2}\right) \longrightarrow \ldots \quad \longrightarrow \quad(A, i)
$$

with infinite fibers and

$$
\operatorname{Vol}_{b_{0}}\left(B_{l}, j_{l}\right) \longrightarrow \operatorname{Vol}_{a_{0}}(A, i)<\infty, \text { as } l \longrightarrow \infty
$$

(For $q=2$, our covering $p:\left(B_{0}, j_{0}\right) \longrightarrow(A, i)$ has branches off the base-ray of $\left(B_{0}, j_{0}\right)$ only at odd vertices $b_{2 l-1}, l=1,2, \ldots$, since our method for constructing such coverings relies on a sequence of vertices $\left\{a_{l}\right\}$ in $V A$ with $q_{l+1}-1>2 q_{l}$, and this is not true for consecutive terms of the sequence $a_{0}, a_{1}, \ldots$ when $q=2$.)

We have the following: 
(4.1) Theorem. Let $(A, i)$ be a parabolic lattice tree. If $(A, i)$ has a covering $\tilde{p}:(\tilde{B}, \tilde{j}) \longrightarrow(A, i)$ with infinite fibers and finite volume, then $(A, i)$ has an infinite sequence of coverings:

$$
\left(B_{0}, j_{0}\right) \stackrel{p_{0}}{\longrightarrow}\left(B_{1}, j_{1}\right) \stackrel{p_{1}}{\longrightarrow}\left(B_{2}, j_{2}\right) \stackrel{p_{2}}{\longrightarrow} \ldots \quad \longrightarrow \quad(A, i)
$$

with infinite fibers, and there exists $a_{0} \in V A, b_{l} \in V B_{l}$ with $p_{0}\left(b_{l}\right)=a_{0}$ for $l=1,2, \ldots$ such that

$$
\operatorname{Vol}_{b_{l}}\left(B_{l}, j_{l}\right) \longrightarrow \operatorname{Vol}_{a_{0}}(A, i) \quad<\infty, \text { as } l \longrightarrow \infty .
$$

Hence we obtain an infinite ascending chain of closed subgroups of $A u t(X)$ :

$$
G_{\left(B_{0}, j_{0}\right)} \leq G_{\left(B_{1}, j_{1}\right)} \leq G_{\left(B_{2}, j_{2}\right)} \leq \cdots \leq G_{(A, i)},
$$

(with notation as in (1.5)), and non-uniform $G_{(A, i)}$-lattices $\Gamma_{l}$ with $\Gamma_{l} \leq G_{\left(B_{l}, j_{l}\right)}$, $l=0,1,2 \ldots$

Proof. Fix a terminal vertex $a_{0} \in V A$, and let $\left(e_{1}, e_{2}, \ldots\right)$ be the unique path from $a_{0}$ to the end of $(A, i)$, a base-ray for $(A, i)$, denoted $\left(A^{\prime}, i\right)$. Corollary (3.8) shows that a covering $\tilde{p}:(\tilde{B}, \tilde{j}) \longrightarrow(A, i)$ with infinite fibers and finite volume induces a 'decoration' $(B, j)$ of a covering $p^{\prime}:\left(B^{\prime}, j^{\prime}\right) \longrightarrow\left(A^{\prime}, i\right)$ with infinite fibers and finite volume of the base-ray $\left(A^{\prime}, i\right)$.

Let $\left(\tilde{B}^{\prime}, \tilde{j}^{\prime}\right)$ be the canonical reduction of $\left(B^{\prime}, j^{\prime}\right)$ as in Theorem $(2.8)$, and $\operatorname{set}\left(B_{0}^{\prime}, j^{\prime}\right)=$ $\left(\tilde{B}^{\prime}, \tilde{j}^{\prime}\right)$. Let $\left(b_{0}^{\prime}, b_{1}^{\prime}, \ldots\right)$ denote the vertex sequence of the base-ray of $\left(B_{0}^{\prime}, j_{0}^{\prime}\right)$. Suppose that $\left(B_{0}^{\prime}, j_{0}^{\prime}\right)$ has branches $B_{k}^{\prime}, B_{k+n_{0}}^{\prime}, B_{k+n_{1}}^{\prime} \ldots$, as defined in Section 2, (2), (3), (4), at vertices $b_{k}^{\prime}, b_{k+n_{0}}^{\prime}, b_{k+n_{1}}^{\prime} \ldots$ along the base-ray of $\left(B_{0}^{\prime}, j_{0}^{\prime}\right)$ :

$\left(B_{0}^{\prime}, j_{0}^{\prime}\right)=$

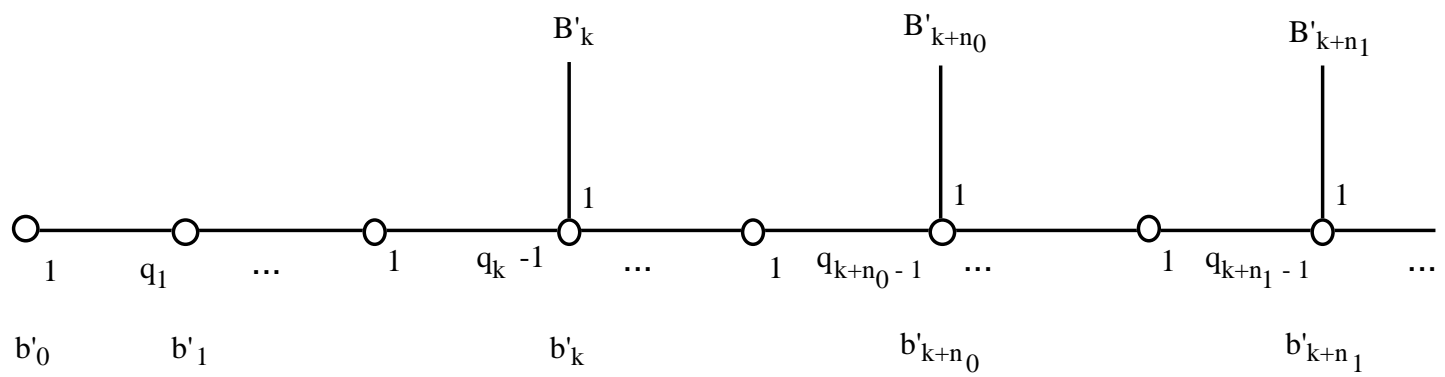

We define the covering $p_{0}^{\prime}:\left(B_{0}^{\prime}, j_{0}^{\prime}\right) \longrightarrow\left(B_{1}^{\prime}, j_{1}^{\prime}\right)$ by 'collapsing' the branch $B_{k}^{\prime}$ onto the base-ray: 
$\left(B_{1}^{\prime}, j_{1}^{\prime}\right)=$

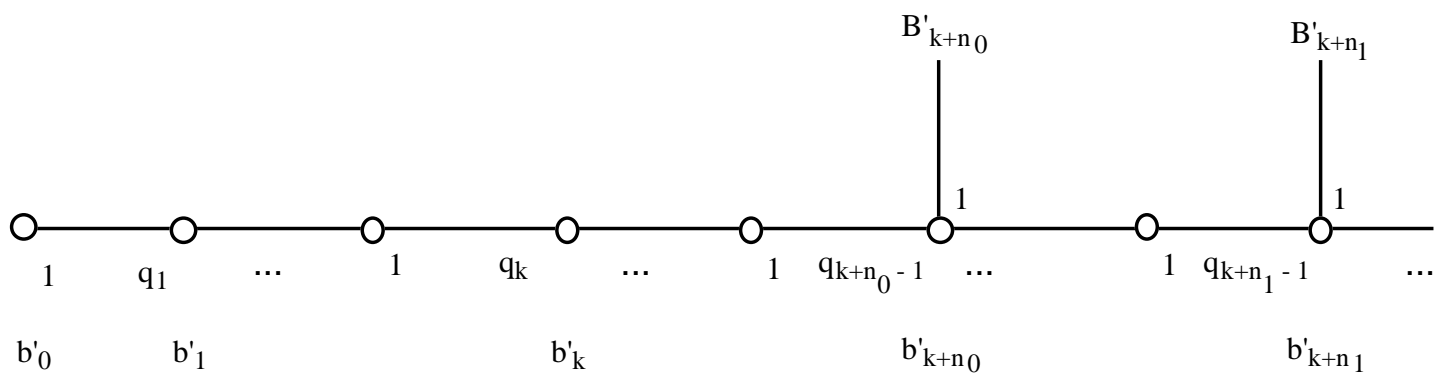

We define an infinite sequence of coverings $p_{l-1}^{\prime}:\left(B_{l-1}^{\prime}, j_{l-1}^{\prime}\right) \longrightarrow\left(B_{l}^{\prime}, j_{l}^{\prime}\right)$, for $l=$ $2,3, \ldots$ by collapsing branches $B_{k+n_{l-2}}^{\prime}$ onto the base-ray. Since $\left(B_{l-1}^{\prime}, j_{l-1}^{\prime}\right)$ has infinite fibers, $\left(B_{l}^{\prime}, j_{l}^{\prime}\right)$ has infinite fibers, for each $l=1,2, \ldots$.

Moreover, it is clear that

$$
\operatorname{Vol}_{b_{0}^{\prime}}\left(B_{l}^{\prime}, j^{\prime}\right) \longrightarrow \operatorname{Vol}_{a_{0}}\left(A^{\prime}, i\right)<\infty, \quad \text { as } l \longrightarrow \infty .
$$

By 'decorating' the sequence of coverings $p_{l-1}^{\prime}:\left(B_{l-1}^{\prime}, j_{l-1}^{\prime}\right) \longrightarrow\left(B_{l}^{\prime}, j_{l}^{\prime}\right)$, for $l=2,3, \ldots$ with finite subtrees, as in (3.2), we obtain a sequence of coverings

$$
\left(B_{0}, j_{0}\right) \stackrel{p_{0}}{\longrightarrow}\left(B_{1}, j_{1}\right) \stackrel{p_{1}}{\longrightarrow}\left(B_{2}, j_{2}\right) \stackrel{p_{2}}{\longrightarrow} \ldots \quad \longrightarrow \quad(A, i)
$$

with infinite fibers, and it follows easily that

$$
\operatorname{Vol}_{b_{0}^{\prime}}\left(B_{l}, j_{l}\right) \longrightarrow \operatorname{Vol}_{a_{0}}(A, i)<\infty, \quad \text { as } l \longrightarrow \infty .
$$

The existence of the infinite ascending chain of closed subgroups of $\operatorname{Aut}(X)$

$$
G_{\left(B_{0}, j_{0}\right)} \leq G_{\left(B_{1}, j_{1}\right)} \leq G_{\left(B_{2}, j_{2}\right)} \leq \ldots \leq G_{(A, i)}
$$

follows from (1.6) (a).

Finally, each $\left(B_{l}, j_{l}\right)$ admits a finite faithful grouping, denoted $\mathbb{B}_{l}, l=0,1,2, \ldots$ which gives rise to a non-uniform lattice

$$
\Gamma_{l}=\pi_{1}\left(\mathbb{B}_{l}, b_{l}\right) \leq G_{\left(B_{l}, j_{l}\right)}, \quad l=0,1,2, \ldots,
$$

for $b_{l} \in V B_{l}, l=0,1,2, \ldots$. 
(4.2) Question. Let $(A, i)$ be a parabolic lattice ray with unbounded index, let $p^{\prime}:\left(B^{\prime}, j^{\prime}\right) \longrightarrow(A, i)$ be a covering with infinite fibers and finite volume, and let $(B, j)$ be the canonical reduction of $\left(B^{\prime}, j^{\prime}\right)$ as in Theorem (2.8):

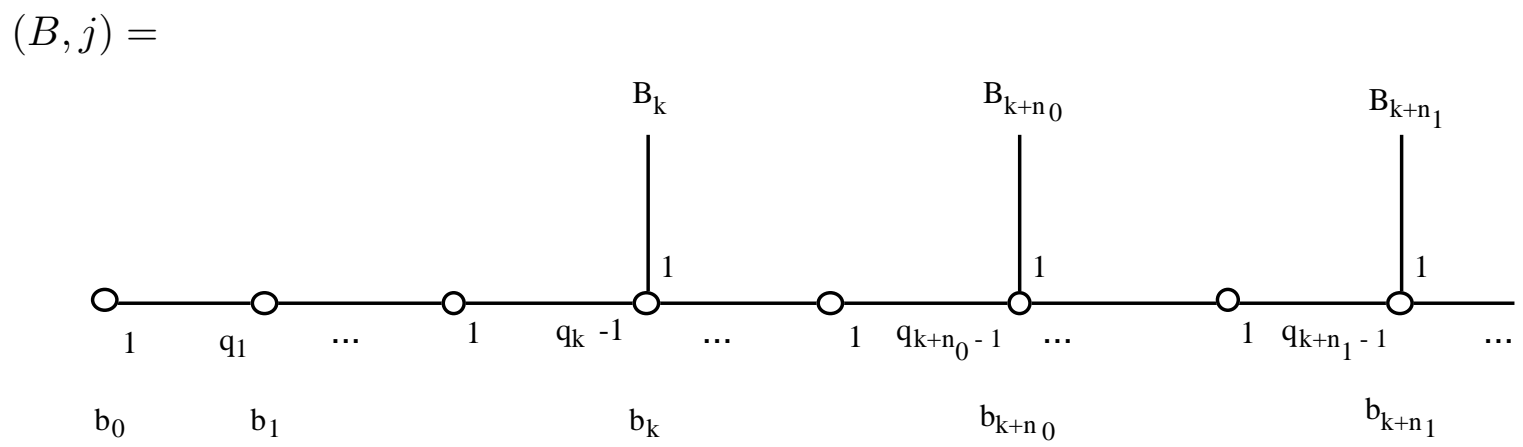

For $l=1,2, \ldots$ let $p_{l-1}:\left(B_{l-1}, j_{l-1}\right) \longrightarrow\left(B_{l}, j_{l}\right)$ be the sequence of coverings obtained in Theorem (4.1), with $\left(B_{0}, j_{0}\right)=(B, j)$, and let $G_{\left(B_{l-1}, j_{l-1}\right)}$ be the corresponding sequence of closed subgroups of $A u t(X)$, for $X=\widetilde{(A, i)}$. Is it true that

$$
G_{\left(B_{l}, j_{l}\right)}=G_{\left(B_{(l-1)}, j_{l-1}\right)} \rtimes \operatorname{Aut}\left(T_{k+n_{l-2}}\right)
$$

where $T_{k+n_{l-2}}$ is a lifting to $X$ of the branch $B_{k+n_{l-2}}$ at the vertex $b_{k+n_{l-2}}$ (with $n_{-1}=$ $0)$ ?

\section{REFERENCES}

[BCR] Bass, H, Carbone L, and Rosenberg, G, The existence theorem for tree lattices, Appendix B, 'Tree Lattices' by Hyman Bass and Alex Lubotzky (2000), Progress in Mathematics, 176 Birkhauser, Boston.

[BK] Bass H and Kulkarni R, Uniform tree lattices, Journal of the Amer Math Society, vol. 3 no. 4 (1990).

[BL] Bass H and Lubotzky A, Tree lattices, Progress in Mathematics, 176 Birkhauser, Boston (2000).

[BT] Bass $\mathrm{H}$ and Tits J, A discreteness criterion for certain tree automorphism groups, Appendix [BT], to 'Tree Lattices' by Hyman Bass and Alex Lubotzky (2000), Progress in Mathematics, 176 Birkhauser, Boston.

[C1] Carbone, L, Non-uniform lattices on uniform trees, Memoirs of the AMS, vol. 152, no. 724 (July 2001).

[C2] Carbone, L, Non-minimal tree actions and the existence of non-uniform tree lattices, (Preprint) (2001).

[CE] Carbone, L and Enos, J, Non-uniform coverings of edge-indexed trees, Report on undergraduate research experience at Harvard University, funded by VIGRE (2000).

[CR] Carbone, L and Rosenberg, G, Lattices on non-uniform trees, Submitted (2001).

[R] Rosenberg, G, Infinite towers of uniform tree lattices, PhD. Thesis, Columbia University (2001).

Department of Mathematics, Harvard University, Science Center 325, One Oxford Street, Cambridge, MA 02138

e-mail: lisa@math.harvard.edu

Department of Mathematics, Harvard University, Science Center 325, One Oxford Street, Cambridge, MA 02138

e-mail: dclark@fas.harvard.edu 